\title{
Stable source connection and assignment problems as multi- period shortest path problems
}

\author{
Leanne Streekstra (Budapest University of Technology and Economics) \\ Christian Trudeau (University of Windsor)
}

Working paper 20-03

Second version - 2022

Working papers are in draft form. This working paper is distributed for purposes of comment and discussion only. It may not be reproduced without permission of the copyright holder. Copies of working papers are available from the author or at http://ideas.repec.org/s/wis/wpaper.html. 


\title{
Stable source connection and assignment problems as multi-period shortest path problems*
}

\author{
Leanne Streekstra ${ }^{\dagger} \quad$ Christian Trudeau ${ }^{\ddagger}$
}

August 14, 2022

\begin{abstract}
We extend the familiar shortest path problem by supposing that agents have demands over multiple periods. This potentially allows agents to combine their paths if their demands are complementary; for instance if one agent only needs a connection to the source in the summer while the other requires it only in the winter.

We not only show that the resulting cost sharing problem always generates a totally balanced game, regardless of the number of agents and periods, the cost structure or the demand profile, but that all totally balanced games are representable as MSP problems.

We then exploit the fact that the model encompasses many well-studied problems to obtain or reobtain balancedness and total-balancedness results for source-connection problems, market problems and minimum coloring problems.

JEL classification numbers: C71, D63.
\end{abstract}

Keywords: shortest path, demand over multiple periods, cooperative game, core, total-balancedness, source-connection, assignment.

\section{Introduction}

Shortest path problems are well-studied in operations research and economics. While they are often used to determine, for instance, the quickest route for a truck making a delivery from A to $\mathrm{B}$, we are interested in applications in which capacity has to be built to connect agents to a source, the capacity is not easily adjustable and the cost is linearly increasing with capacity. Gas and oil

\footnotetext{
${ }^{*}$ Christian Trudeau acknowledges financial support by the Social Sciences and Humanities Research Council of Canada [grant number 435-2019-0141].

${ }^{\dagger}$ Research Centre of QSMS, Faculty of Economic and Social Sciences, Budapest University of Technology and Economics, Megyetem rkp. 3., H-1111 Budapest, Hungary. Email: leanne.streekstra@outlook.com

${ }^{\ddagger}$ Department of Economics, University of Windsor, 401 Sunset Avenue, Windsor, Ontario, Canada. Email: trudeauc@uwindsor.ca
} 
pipelines, as well as rail networks, are some examples that fit the bill. Following Rosenthal (2013) and Bahel and Trudeau (2014), we are interested in the cost sharing problem generated by these situations. We, however, generalize the problem by supposing that there are multiple periods, and that agents have demands that vary over these periods. Imagine two small cities, A and B, located respectively to the northeast and southeast of the capital city. City A is a ski destination popular in the winter, while city B is a beach destination popular in the summer. When designing a rail network that will connect both cities to the capital, we could connect them both directly to the capital, but it probably makes more sense to connect only one, say A, while connecting B to A, to take advantage of the complementary demand to both cities. We could say the same of gas pipelines to cities $\mathrm{A}$ and $\mathrm{B}$, for which the gas is either used for heating in the winter or cooling in the summer.

The model in itself, that we call the multi-period shortest path (MSP) problem, ${ }^{1}$ has not yet been studied in economics, and our first contribution is to show that the core of the resulting cooperative game is always non-empty. Thus, we are always able to share the cost in a way that makes sure that no group of agents could jointly connect to the source at a cost cheaper than the amount they are assigned. The non-vacuity of the core thus extends from the classic, one-period shortest path problem (Rosenthal (2013)), and does not require any condition on the network structure, number of periods, number of agents or demand profile. Our proof is constructive, as we provide a core allocation built from the shadow prices of the constraints of our problem, expressed as a linear program (Theorem 1).

In fact, the same reasoning applies to any subgame as well, making the games totally-balanced. Furthermore, we show that any totally balanced game has an MSP representation, once appropriately zero-normalized and adjusted from value game to cost game (Theorem 2).

We can extend our result that MSP problems generate games with non-empty cores in various ways. Notably, it is valid whether we suppose that a coalition of agents can connect through (noncooperating) neighbors or not (Corollary 2). These variants are discussed for the related minimum cost spanning tree problem in Trudeau and Vidal-Puga (2019). Our non-vacuity result also extends to private games (agents can prevent others from using their nodes) where the cost functions on all edges are convex, if we do not have unoccupied (Steiner) nodes (Theorem 4). That proof is strongly inspired by Quant et al. (2006), who study a (one-period) network flow problem where on each edge of the network there is a cost function that is convex with respect to the flow. The result

\footnotetext{
${ }^{1}$ Finding a shortest path is optimal when we are looking for the cheapest way to deliver goods to agents whose demands are over a single period and cost is linearly increasing with the flow on each edge. When we extend the demand profiles to multiple periods, the resulting optimal way to connect agents to the source might not be a shortest path in any period, see Example 1. However, the name shortest path is associated with the problem itself, and it is what we extend to multiple periods and call MSP, even though the solution concept to the operations research problem does not extend.
} 
also extends to cases where agents can own multiple nodes (Theorem 5).

The equivalence between games generated by MSP problems and totally-balanced games is similar to the result of Shapley and Shubik (1969) and Kalai and Zemel (1982), who obtain an equivalence between totally-balanced games and, respectively, market games and so-called games of flow. ${ }^{2}$ In our opinion, our result is a valuable addition as MSP problems naturally encompass, in full or in part, a variety of well-studied problems. This allows us to obtain or reobtain results on balancedness and total-balancedness, as well as draw new links between various sets of problems. To do so, we provide MSP representations that are built directly from the parameters of the problem, instead of from the values of the corresponding game as in Theorem 2. The games that we describe are based on operation research problems. While the result in Theorem 2 allows us to build the link to MSP problems using post-optimization, totally-balanced coalitional games constructed from operation research problem, in Section 4 we establish direct links between MSP problems and various pre-optimization operations research problems. Finding the optimal network in the MSP problem is then equivalent to finding the optimal solution in the corresponding operations research problem. These pre-optimization links allow for a better understanding of the links between the various sets of problems.

Source-connection problems are an obvious group of problems that can be rewritten as MSP problems, such as the aforementioned (one-period) shortest path and the minimum cost spanning tree (mcst) problems (Bird (1976)). Mcst problems are such that on any edge the first unit of capacity is costly, but others are free. We also provide results on airport and irrigation problems (Aadland and Kolpin, 1998; Thomson, 2007).

Less obviously, MSP problems also encompass assignment problems (Shapley and Shubik (1971)), where we need to match agents belonging to different sides of the market, with the classic example being a housing market. As in the example described above, we can construct an MSP problem in which we have some cities demanding in the summer and some others in the winter, and matching them allows to save on cost. It is well-known that the core of an assignment game is always nonempty, and we show that all assignment problems can be written as an MSP problem. Extensions of the assignment problem to $m$-sides, with $m>2$, is where the connection to MSP problems is more useful. Many variants have been proposed and we study the strict $m$-sided assignment problem of Quint (1991) and the generalized version of Atay et al. (2016). In the former, value is created only when we match a group that contains one player from each side, while in the latter there is also some value created when we match smaller groups of agents from different sides. In both cases, the core of the corresponding game can be empty, and sufficient conditions for its non-vacuity have been proposed. Some but not all of these $m$-sided assignment problems have a corresponding MSP

\footnotetext{
${ }^{2}$ Similar results exist for different types of games. Rosenmüller (1981) provides an equivalence between convex games and production games, while Van den Nouweland et al. (1993) show the equivalence between monotonic games and so-called spanning network games.
} 
representation, but by our stability result, if an assignment problem can be represented as an MSP problem, it has a non-empty core. We provide sufficient conditions for representability as MSP problems, and thus for totally-balancedness, extending a condition provided by Stuart Jr (1997).

We also examine compatibility problems, most notably the minimum coloring problem, studied as a cost sharing problem by Okamoto (2008). In those problems, the set of agents has to be partitioned in groups, such that agents in the same group have no conflict with each other. The conflicts are represented in a graph, with an edge between $i$ and $j$ on the graph indicating that $i$ and $j$ are in conflict and cannot be assigned to the same group. A sufficient condition for the nonvacuity of the core is provided, requiring that for any subset of agents, the number of groups needed to avoid conflicts (the chromatic number of the graph) is equal to the size of the largest group of agents all in conflict with each other (the size of the largest clique in the graph). A simpler version where the condition is always satisfied is presented by Bahel and Trudeau (2019) where agents have time-sensitive jobs to be processed on a machine, and in which we are trying to determine the smallest number of machines required to process all jobs without conflict. We show that when the condition of Okamoto (2008) is satisfied, we have representability as an MSP problem. We are also able to use our stability result in a different way to extend the set of problems with non-empty cores. If we weaken the condition of Okamoto (2008) so that it only needs to hold for the grand coalition, then there exists an MSP problem which has the same cost for the grand coalition and a cost that might be smaller but not strictly larger for any other coalition. The core of this MSP problem is thus a subset of the core of the minimum coloring problem. By our non-vacuity result for the MSP problem, the core of the minimum coloring problem is then also non-empty.

Comparing how we have represented these various problems as MSP problems allows us to draw new links between these problems. One such example is that it becomes clear that mcst problems are $m$-sided assignment games with a single agent per side, allowing us to find a new sufficient condition for $m$-sided assignment problems to have a non-empty core (Theorem 8 ). Comparing $m$-sided assignment problems to compatibility problems also allows us to see that they are both extensions of classic (2-sided) assignment problems.

The rest of the paper is divided as follows. Section 2 describes the MSP problem and the associated cooperative game. Section 3 is devoted to the non-emptiness of the core. In Section 4 we show applications to source connection, market and compatibility problems.

\section{The model}

Let $N$ be the set of agents. Let $L \supseteq N$ be the set of nodes, with $|L|$ finite. Nodes in $L \backslash N$ are unoccupied, and are called Steiner nodes. For all $S \subseteq N$, let $\bar{S}=S \cup(L \backslash N)$ be the set of nodes occupied by agents in $S$ together with the Steiner nodes. Let $M=\{1, \ldots, m\}$ be the set of periods, with $m$ finite. We suppose that $|N| \geq 2$ and $m \geq 1$. 
Nodes are located at different points in space. We represent their position on a complete, simple digraph, with each agent occupying a different node. There is a special node, 0 , that we call the source. For all $S \subseteq L, S_{0}:=S \cup\{0\}$. Let $E:=\left\{(i, j) \mid i, j \in L_{0}\right.$ and $\left.i \neq j\right\}$ denote the set of directed edges. For any $S \subset L$, let $E^{S}:=\left\{(i, j) \mid i, j \in S_{0}\right.$ and $\left.i \neq j\right\}$.

For all $i \in N$, let $q_{i}=\left(q_{i 1}, \ldots, q_{i m}\right) \in \mathbb{R}_{+}^{M}$ be the demand profile for agent $i$. In particular, for $t \in M, q_{i t}$ is the demand of agent $i$ at time $t$. Let $Q=\left\{q_{i}\right\}_{i \in N}$ be the demand profiles for all agents. For all $t \in M$, let $N^{t}(Q)=\left\{i \in N \mid q_{i t}>0\right\}$ be the set of agents with a strictly positive demand at time $t$. Steiner nodes have no demand, but it is often convenient to define the demand profile on $L$. Let $\bar{Q}=\left\{\bar{q}_{i}\right\}_{i \in L}$ be such that $\bar{q}_{i}=q_{i}$ for all $i \in N$ and $\bar{q}_{i}=0^{M}$ for all $i \in L \backslash N$. When $|M|=|N|$ we sometimes use a "diagonal matrix" demand $D^{|N|}$, such that $D_{i t}^{|N|}=1$ if $i=t$ and $D_{i t}^{|N|}=0$ otherwise.

To obtain their demand, agents need to build paths to the source. In addition, the capacity of these paths must be large enough to carry their demands in every period. For each edge $(i, j) \in E$, $c_{i j} \geq 0$ represents the cost to install a capacity of one unit from $i$ to $j$. Cost is linear with capacity, so for any $a \geq 0$, the cost of installing a capacity of $a$ on edge $(i, j)$ is $a \cdot c_{i j}$. Let $c=\left(c_{i j}\right)_{(i, j) \in E}$ be the collection of costs of all edges. We abuse language slightly by calling it a cost matrix. We sometimes make assumptions on $c$. We say that $c$ is symmetric if $c_{i j}=c_{j i}$ for all $i, j \in L_{0}$. We say that $c$ satisfies the triangle inequality if for any $i, j, k \in L_{0}, c_{i k} \leq c_{i j}+c_{j k}$.

The tuple $(N, L, M, Q, c)$ is called a multi-period shortest path problem (MSP problem for short). When $N=L$ we sometimes write $(N, M, Q, c)$ instead of $(N, L, M, Q, c)$.

\subsection{Optimal networks}

The first objective is to build a network connecting all agents to the source that contains enough capacity to simultaneously carry all demands at each period. For any $(i, j) \in E$, let $z_{i j} \geq 0$ be the capacity installed from $i$ to $j$. Let $z=\left(z_{i j}\right)_{(i, j) \in E}$ be the collection of capacities on all edges. We call $z$ a network. For any $(i, j) \in E$ and any $t \in M$, let $x_{i j t}$ be the flow sent from $i$ to $j$ at period $t$.

The problem of finding the minimum cost to provide the demand to our set of agents can be written as a linear program $\gamma(N, L, M, Q, c)$ : 


$$
\begin{aligned}
& \min \sum_{(j, k) \in E} z_{j k} c_{j k} \\
& \text { s.t. : } \\
& Z_{j k t} \quad: \quad z_{j k} \geq x_{j k t} \text { for all }(j, k) \in E, t \in M \\
& O_{t} \quad: \quad \sum_{i \in L} x_{i 0 t} \geq \sum_{i \in L} \bar{q}_{i t} \text { for all } t \in M \\
& I_{i t} \quad: \quad x_{i 0 t}+\sum_{j \in L \backslash i}\left(x_{i j t}-x_{j i t}\right) \geq \bar{q}_{i t} \text { for all } i \in L, t \in M \\
& z_{j k} \geq 0, x_{j k t} \geq 0 \text { for all }(j, k) \in E, t \in M
\end{aligned}
$$

In words, constraints $Z_{j k t}$ guarantee that the flow is no larger than the capacity for any edge and any period. Constraints $O_{t}$ ensure that in any period the flow coming into the source is enough to serve the demand of all agents in that period. Constraints $I_{i t}$ guarantee that for any node and any period, outgoing flows minus incoming flows is at least as large as the demand of the agent (if any) at that node in that period. Since the demand of a Steiner node is 0 in each period, this also ensures that the outgoing flow from a Steiner node is at least as large as the incoming flow.

A solution to the linear program is $z^{*}=\left(z_{j k}^{*}\right)_{(j, k) \in E}, x^{*}=\left(x_{j k t}^{*}\right)_{\substack{j, k) \in E \\ t \in M}}$. We call a network that satisfies all the constraints, but is not necessarily the cheapest, feasible.

Let $P_{Z_{j k t}}$ be the shadow price associated with $Z_{j k t}, P_{O_{t}}$ be the shadow price associated with constraint $O_{t}$ and $P_{I_{i t}}$ be the shadow price associated with constraint $I_{i t}$.

The dual is the problem $\delta(N, L, M, Q, c)$ :

$$
\begin{aligned}
& \max \sum_{t \in M}\left(P_{O_{t}} \sum_{i \in L} \bar{q}_{i t}\right)+\sum_{t \in M} \sum_{i \in L} \bar{q}_{i t} P_{I_{i t}} \\
& \text { s.t. } \quad: \\
& C_{j k} \quad: \quad \sum_{t \in M} P_{Z_{j k t}} \leq c_{j k} \text { for all }(j, k) \in E \\
& A_{i t}: \quad P_{O_{t}}+P_{I_{i t}}-P_{Z_{i 0 t}} \leq 0 \text { for all } i \in L, t \in M \\
& B_{i j t}: \quad P_{I_{i t}}-P_{I_{j t}}-P_{Z_{i j t}} \leq 0 \text { for all } i \in L, j \in L \backslash i, t \in M \\
& P_{Z_{j k t}} \geq \quad 0, P_{O_{t}} \geq 0, P_{I_{i t}} \geq 0 \text { for all }(j, k) \in E, i \in L, t \in M
\end{aligned}
$$

A solution to the dual is $P_{Z}^{*}=\left(P_{Z_{j k t}}^{*}\right)_{\substack{(j, k) \in E \\ t \in M}}, P_{O}^{*}=\left(P_{O_{t}}^{*}\right)_{t \in M}, P_{I}^{*}=\left(P_{I_{i t}}^{*}\right)_{\substack{i \in L \\ t \in M}}$.

Let $C(N, Q)=\sum_{(j, k) \in E} z_{j k}^{*} c_{j k}=\sum_{t \in M}\left(P_{O_{t}}^{*} \sum_{i \in L} \bar{q}_{i t}\right)+\sum_{t \in M} \sum_{i \in L} \bar{q}_{i t} P_{I_{i t}}^{*}$ be the minimal cost to serve the grand coalition $N$. In the same manner, for all $S \subseteq N$, we define as $\gamma\left(N, L, M, Q^{S}, c\right)$ the problem in which only agents in $S$ are demanding, and $C(S, Q)$ its minimal cost, where $Q^{S}$ is the restriction of $Q$ to $S$, i.e. for all $t \in M, q_{i t}^{S}=q_{i t}$ if $i \in S$ and $q_{i t}^{S}=0$ otherwise. When there is no confusion we write $C(N)$ instead of $C(N, Q)$ and $C(S)$ instead of $C(S, Q)$. 
Example 1 Throughout the paper we will illustrate our results with a 4-player example, with $L \backslash N=s_{1}$, and the cost matrix described in Figure 4. Circled numbers represent the agents and the source, whereas $s_{1}$ is a Steiner node. We suppose that the cost matrix is symmetric and the number on an edge $\{i, j\}$ represents the cost from $i$ to $j$ and $j$ to $i$. It is easy to see that the example satisfies the triangle inequality.

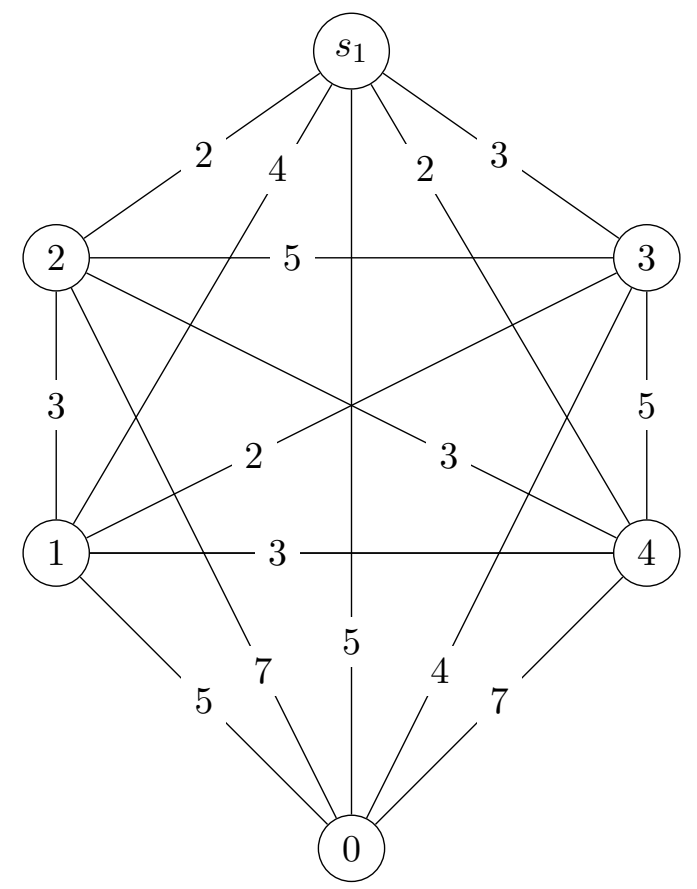

Figure 1: Example of a cost matrix with 4 agents and 1 Steiner node.

Suppose that $m=2$ and that $Q=((1,0),(2,1),(0,1),(2,3))$. While there are 10 units being consumed, they are evenly split between the two periods. Consider the following networks:

$\begin{array}{lllllllllllllll} & z_{10}^{i} & z_{20}^{i} & z_{30}^{i} & z_{40}^{i} & z_{s_{1} 0}^{i} & z_{13}^{i} & z_{41}^{i} & z_{24}^{i} & z_{23}^{i} & z_{1 s_{1}}^{i} & z_{2 s_{1}}^{i} & z_{3 s_{1}}^{i} & z_{4 s_{1}}^{i} & \text { Cost } \\ z^{1} & 1 & 2 & 1 & 3 & 0 & 0 & 0 & 0 & 0 & 0 & 0 & 0 & 0 & 44 \\ z^{2} & 1 & 1 & 1 & 2 & 0 & 0 & 1 & 0 & 1 & 0 & 0 & 0 & 0 & 38 \\ z^{3} & 0 & 0 & 1 & 0 & 4 & 1 & 0 & 0 & 0 & 0 & 2 & 0 & 3 & 36\end{array}$

Network $z^{1}$ connects all agents directly to the source, with a capacity equal to their largest demand over all periods. $z^{2}$ takes advantage of the fact that agents 1 and 4 jointly demand 3 units in each period. Thus, while agent 4 still installs 2 units of capacity on $(4,0)$, he now sends his remaining demand over $(4,1)$, consuming the unused unit of agent 1 in period 2. We do something similar for agents 2 and 3, who together demand 2 units in each period. Each connects to the source with a capacity of 1 and agent 2 sends his remaining demand via agent 3 over $(2,3)$. $z^{3}$ follows the same idea, but instead has agents 1 and 3 and agents 2 and 4 partner up. In this case it is optimal for agents 2 and 4 to connect via the Steiner node, rather than to connect directly to each other. It 
can be verified that $z^{3}$ solves $\gamma(N, L, M, Q, c)$. Thus, $C(N)=36$.

\subsection{Cooperative game}

Let $(N, L, M, Q, c)$ be an MSP problem. Note that $C(\cdot)$ can be regarded as the characteristic function of a cooperative cost game $(N, C)$, describing the cheapest way for any $S \subseteq N$ to obtain its demand. By definition $C(\emptyset)=0$ and $S \subseteq T$ implies that $C(S) \leq C(T)$. For each $S \subseteq N$ we define the subgame $C_{S}$ such that for all $T \in 2^{S}, C_{S}(T) \equiv C(T)$.

An allocation is a vector $y \in \mathbb{R}^{N}$ such that $\sum_{i \in N} y_{i}=C(N)$. For any $S \subseteq N$, we define $y(S) \equiv \sum_{i \in S} y_{i}$. We say that an allocation $y$ is a core allocation if $y(S) \leq C(S)$ for all $S \subset N$. For a cooperative game with characteristic function $C$, the set of all core allocations is denoted with Core $(C)$.

A map $\lambda: 2^{N} \backslash\{\emptyset\} \rightarrow[0,1]$ is said to be balanced if for all $i \in N, \sum_{\substack{S \in 2^{N} \\ i \in S}} \lambda_{S}=1$. Bondareva (1963) and Shapley (1967) have shown that the core of the cooperative (cost) game $(N, C)$ is nonempty if and only if for any balanced map $\lambda, \sum_{S \in 2^{N} \backslash\{\emptyset\}} \lambda_{S} C(S) \geq C(N)$, i.e. if $(N, C)$ is balanced. We say that a game is totally balanced if all subgames have a non-empty core.

We often convert $(N, C)$ into a savings game $(N, V)$ as follows: for any $C(\cdot)$ and $S \subseteq N$, let $V(S)=\sum_{i \in S} C(\{i\})-C(S) . V(S)$ is the savings generated by the cooperation of agents in $S$, compared to each acting on their own. The allocation $y^{v}$ is the savings game-equivalent of $y$ and is defined as follows: for all $i \in N, y_{i}^{v}=C(\{i\})-y_{i}$. The allocation $y^{v}$ is a core allocation if $y^{v}(S) \geq V(S)$ for all $S \subset N$. By definition, $y^{v} \in \operatorname{Core}(V)$ if and only if $y \in \operatorname{Core}(C)$.

Savings games are special cases of value games, which are such that for all $S \subseteq N V(S) \geq 0$ represents the value generated by $S$. For such a game $V$ its zero normalization is $V^{0}$ such that $V^{0}(S)=V(S)-\sum_{i \in S} V(i)$. The restriction from the full set of value games to the set of zeronormalized ones is not consequential. The core of one problem is the translation of the core of the other problem. Notice that our savings games are zero-normalized games. Let $\mathcal{V}^{0}$ be the set of zero-normalized value games.

\section{The core of MSP problems}

\subsection{MSP problems generate totally balanced games}

We show in this section that the core of an MSP game is always non-empty. This is done by showing that the shadow prices of the linear program yield a core allocation. We begin by simplifying the dual problem.

Lemma 1 Let $\mathcal{S}=\left(\left(P_{Z_{i j t}}\right)_{(i, j) \in E, t \in M},\left(P_{O_{t}}\right)_{t \in M},\left(P_{I_{i t}}\right)_{i \in L, t \in M}\right)$ be an optimal solution to the dual and suppose there is some $P_{O_{t^{\prime}}}=\alpha, \alpha>0$. Then solution $\hat{\mathcal{S}}$ which is constructed from $\mathcal{S}$ by replacing $P_{O_{t^{\prime}}}$ with 0 and adding $\alpha$ to $P_{I_{i t^{\prime}}}$ for all $i \in N$ is also an optimal solution to the dual. 
Proof. First, note that $P_{O_{t^{\prime}}}$ only appears in the constraints $A_{i t}$ for $i \in L$, which have the form $P_{O_{t^{\prime}}}+P_{I_{i t^{\prime}}}+P_{Z_{i 0 t^{\prime}}} \leq 0$. It is easy to see that since $\mathcal{S}$ satisfies this constraint, so does $\hat{\mathcal{S}}$.

Next, consider a constraint $B_{i j t^{\prime}}$ for arbitrary $i, j \in L$. As $\mathcal{S}$ satisfies this constraint, we know that $P_{I_{i t^{\prime}}}-P_{I_{j t^{\prime}}}-P_{Z_{i j t^{\prime}}} \leq 0$. But then $\left(P_{I_{i t^{\prime}}}+\alpha\right)-\left(P_{I_{j t^{\prime}}}+\alpha\right)-P_{Z_{i j t^{\prime}}} \leq 0$ is also true and thus $\hat{\mathcal{S}}$ is indeed a solution to the dual.

The value of $\mathcal{S}=\sum_{t \in M}\left(P_{O_{t}} \sum_{i \in L} \bar{q}_{i t}\right)+\sum_{t \in M} \sum_{i \in L} \bar{q}_{i t} P_{I_{i t}}$ differs from $\hat{\mathcal{S}}$ only in

$$
P_{O_{t^{\prime}}} \sum_{i \in L} \bar{q}_{i t^{\prime}}+\sum_{i \in L} \bar{q}_{i t^{\prime}} P_{I_{i t^{\prime}}}=\sum_{i \in L} \bar{q}_{i t^{\prime}} \alpha+\sum_{i \in L} \bar{q}_{i t^{\prime}} P_{I_{i t^{\prime}}}=0+\sum_{i \in L} \bar{q}_{i t^{\prime}}\left(P_{I_{i t^{\prime}}}+\alpha\right)
$$

Hence, the value of $\hat{\mathcal{S}}$ is the same as that of $\mathcal{S}$ and $\hat{\mathcal{S}}$ is thus also an optimal solution.

Lemma 2 Let $\mathcal{S}=\left(\left(P_{Z_{i j t}}\right)_{(i, j) \in E, t \in M},\left(P_{O_{t}}\right)_{t \in M},\left(P_{I_{i t}}\right)_{i \in L, t \in M}\right)$ be an optimal solution to the dual and suppose there is an $i \in L, t \in M$ s.t. $P_{Z_{i 0 t^{\prime}}}>P_{O_{t^{\prime}}}+P_{I_{i t^{\prime}}}$. Then $\overline{\mathcal{S}}$ which is constructed from $\mathcal{S}$ by setting $P_{Z_{i 0 t^{\prime}}}$ equal to $P_{O_{t^{\prime}}}+P_{I_{i t^{\prime}}}$ is also an optimal solution to the dual.

Proof. As $P_{Z_{i 0 t^{\prime}}}$ has no influence on the value of the solution, it suffices to show that $\overline{\mathcal{S}}$ satisfies all the constraints.

$P_{Z_{i 0 t^{\prime}}}$ appears in two constraints: $C_{i 0}$ and $A_{i t^{\prime}}$. As we know that $\sum_{t \in M} P_{Z_{i 0 t}} \leq c_{i 0}$ and $P_{O_{t^{\prime}}}+P_{I_{i t^{\prime}}}<P_{Z_{i 0 t^{\prime}}}$, it follows that $\overline{\mathcal{S}}$ satisfies $C_{i 0}$. Next, consider $A_{i t^{\prime}}: P_{O_{t^{\prime}}}+P_{I_{i t^{\prime}}}-P_{Z_{i 0 t^{\prime}}} \leq 0$. As $\overline{\mathcal{S}}$ is such that $P_{Z_{i 0 t^{\prime}}}=P_{O_{t^{\prime}}}+P_{I_{i t^{\prime}}}$ it follows that $\overline{\mathcal{S}}$ satisfies $A_{i t^{\prime}}$ and moreover, it is binding.

Thus without loss of generality we can assume all $P_{O_{t}}$ to be 0 and $P_{Z_{i 0 t}}=P_{I_{i t}}$ for all $i \in N, t \in$ $M$.

Given these simplifications, we can use the following simplified dual $\delta^{*}(N, L, M, Q, c)$ :

$$
\begin{aligned}
& \max \sum_{t \in M} \sum_{i \in L} \bar{q}_{i t} P_{Z_{i 0 t}} \\
\text { s.t. } & : \\
C_{j k}: & \sum_{t \in M} P_{Z_{j k t}} \leq c_{j k} \text { for all }(j, k) \in E \\
B_{i j t}: & P_{Z_{i 0 t}}-P_{Z_{j 0 t}}-P_{Z_{i j t}} \leq 0 \text { for all } i \in L, j \in L \backslash i, t \in M \\
P_{Z_{j k t}} \geq & 0 \text { for all }(j, k) \in E, t \in M
\end{aligned}
$$

Let $P_{Z}^{*}$ be a solution of the simplified dual. Define $y^{*}\left(P_{Z}^{*}\right)$ as follows. For all $i \in N, y_{i}^{*}\left(P_{Z}^{*}\right)=$ $\sum_{t \in M} \bar{q}_{i t} P_{Z i 0 t}^{*}$

Theorem 1 For all $M S P$ problems $(N, L, M, Q, c)$ let $P_{Z}^{*}$ be a solution of the simplified dual $\delta^{*}(N, L, M, Q, c)$. Then, $y^{*}\left(P_{Z}^{*}\right)$ is a core allocation. 
Proof. First, it is immediate that $\sum_{i \in N} y_{i}^{*}\left(P_{Z}^{*}\right)=C(N, M, Q, c)$, as $\sum_{i \in N} \sum_{t \in M} \bar{q}_{i t} P_{Z_{i 0 t}}^{*}=$ $\sum_{i \in L} \sum_{t \in M} \bar{q}_{i t} P_{Z_{i 0 t}}^{*}$ is the optimal value of the simplified dual, with the equality coming from $\bar{q}_{i t}=0$ for all $i \in L \backslash N$ and all $t \in M$. Thus, $y^{*}\left(P_{Z}^{*}\right)$ is budget balanced.

For $S \subset N$, consider the simplified dual problem $D^{*}\left(N, L, M, Q^{S}, c\right)$ and notice that the constraints are independent of $S$. Therefore, for $P^{*}$ a solution of the simplified dual $\delta^{*}(N, L, M, Q, c)$ we

have that $\sum_{t \in M} \sum_{i \in S} \bar{q}_{i t} P_{Z_{i 0 t}}^{*} \leq C(S)$, as $P^{*}$ is among the set of potential maximizers for coalition $S$. Rearranging, we obtain

$$
\begin{aligned}
C(S) & \geq \sum_{i \in S} \sum_{t \in M} \bar{q}_{i t} P_{Z_{i 0 t}}^{*} \\
& =\sum_{i \in S} y_{i}^{*}\left(P_{Z}^{*}\right)
\end{aligned}
$$

and thus $y^{*}\left(P_{Z}^{*}\right)$ is a core allocation.

MSP problems therefore have the interesting feature that we can always share the cost in a stable manner, guaranteeing that no group has incentives to secede and do the project by itself. While this is known to be true for classic, one-period shortest path problems, it is interesting to see that it also holds in our more general setting, with no conditions on the number of players, number of periods, demands or cost structure.

It is easy to see that for an MSP problem $(N, L, M, Q, c)$ the above proof extends to all subgames, yielding the following corollary.

Corollary 1 For all MSP problems $(N, L, M, Q, c), C(\cdot)$ is totally balanced.

\subsection{Totally balanced games can be represented as MSP problems}

We show that all zero-normalized totally-balanced value games can be represented as an MSP problem.

Theorem 2 Every zero-normalized totally balanced game $V$ can be rewritten as an MSP problem.

Proof. Let $V$ be a zero-normalized totally balanced game. We construct an MSP as follows. Let $|M|=|N|$ and $Q=D^{|N|}$. Let $L=2^{N}$. For all $i \in N$, we write $i \operatorname{instead~of~}\{i\}$.

Let $\kappa=V(N)$. For all $S \in 2^{N}$, let $c_{S 0}=(|S|-1) \kappa$ if $|S|>1$ and $c_{S 0}=\kappa$ otherwise. For all $i \in N$ and $S \supset\{i\}$, set $c_{i S}=(\kappa-V(S)) /|S|$. For any other edge $e \in E$, let $c_{e}=|N| \kappa$.

We now need to show that this MSP problem indeed corresponds to $V$. First note that we may assume that in an optimal network only edges of the type $(S, 0)$ and $(i, S)$ with $i \in S$ have a strictly positive capacity. To see this consider the following. Suppose there is an edge $(S, i)$ (or $(S, T)$ ) with strictly positive capacity $z_{S i}$. The cost associated with this edge is then $z_{S i} \cdot|N| x$. Let $T \subseteq N$ be the set of agents who send (part of) their flow over $(S, i)$. Then we can set $z_{S i}=0$ and increase 
$(j, 0)$ by $z_{S i}$ for all $j \in T$ and we would still have a feasible network. The difference between the cost of this new network and the original one is $z_{S i} \cdot|T| \kappa-z_{S i} \cdot|N| \kappa \leq 0$.

Now suppose there is an edge $(i, S)$, with $i \notin S$ with a strictly positive capacity $z_{i S}$. In this case, we can replace $z_{i S}$ with $z_{i 0}$. This new network has a lower cost than the old one as $(i, S)$ has a cost of $|N| \kappa$ and $(i, 0)$ has a cost of $\kappa$. Moreover, it is still feasible as all flows that went through $(i, S)$ previously, can now be redirected through $(i, 0)$ instead. Note that we can always assume that edges leaving the source have a capacity of 0 .

Hence we can assume that in an optimal network that for any $i \in N$ only edges $(i, 0),(i, S)$ for $i \in S$ and $(S, 0)$ can have a strictly positive capacity. We will now show by induction on $|S|$ that $\Sigma_{i \in S} c(\{i\})-c(S)=V(S)$ for all $S \subseteq N$. For any $i \in N$, by definition $c(\{i\})-c(\{i\})=0=V(\{i\})$. Note that for any $|S|>1$, if all $i \in S$ connect to $S$ and $S$ is connected to the source with capacity 1 , the total cost is $(|S|-1) \kappa+|S| \frac{\kappa-V(S)}{|S|}=|S| \kappa-V(S)$. Hence $\Sigma_{i \in S} c(\{i\})-c(S)=$ $|S| \kappa-(|S| \kappa-V(S))=V(S)$. Thus, the cost savings generated by $S$ in our MSP problem is at least as large as $V(S)$.

Now take any $S \subseteq N$ and assume that for all $|T|<|S|$ it holds that $\Sigma_{i \in T} c(\{i\})-c(T)=V(T)$ and that the optimal network $z^{T}$ for $T$ consists of a capacity of 1 on just $(T, 0)$ if $|T|=1$ and on $(T, 0)$ as well as $(i, T)$ for all $i \in T$ otherwise. As $c_{T 0}=(|T|-1) \kappa$ and $c_{i 0}=\kappa$ it is easy to see that connecting via $T$ is only beneficial if $z_{i T}=z_{j T} \equiv \alpha_{T}$ for all $i, j \in T$. As a consequence, it is non-optimal to connect via any $T \supset S$. As the flows for any agent need to sum up to 1 , it then follows that the set of potential optimal networks is $\Sigma_{T \subseteq S} \alpha^{T} z^{T}$ for $0 \leq \alpha^{T} \leq 1$ and $\Sigma_{T \ni i} \alpha^{T}=1$ for all $i \in N$.

The cost of such a network is

$$
\begin{aligned}
\sum_{T \subseteq S} \alpha^{T} c(T) & =\sum_{T \subseteq S} \alpha^{T}\left(\sum_{i \in T} c(\{i\})-V(T)\right) \\
& =\sum_{i \in S} c(\{i\})-\sum_{T \subseteq S} \alpha^{T} V(T) \\
& =|S| \kappa-\sum_{T \subseteq S} \alpha^{T} V(T) \\
& \geq|S| \kappa-V(S)
\end{aligned}
$$

and $c(S)=|S| \kappa-V(S)$ as desired. Note that the second equality come from $\left\{\alpha^{T}\right\}_{T \subseteq S}$ being a balanced set of weights, and the inequality comes from $V$ being totally balanced.

It then follows that the optimal network for $S$ is indeed for all agents $i \in S$ to connect via $S$ to the source and hence $\Sigma_{i \in S} c(\{i\})-c(S)=V(S)$.

Combining the previous two results, we obtain the following:

Theorem 3 A game is a zero-normalized totally-balanced (value) game if and only if it is representable as an MSP problem. 


\subsection{Extension to the private property game}

So far, we have made the assumption that agents in $S$ can use the nodes of players in $N \backslash S$ to construct their paths to the source. We can amend this assumption by supposing that a coalition can only use its own nodes, the unoccupied nodes and the source. In many source connection problems, like minimum cost spanning tree problems, these distinct games are called private and public games, depending on whether the nodes are privately or publicly owned. Stated differently, in a private game, an agent can refuse to let others use his node when he is not cooperating with them. Among others, see Trudeau and Vidal-Puga (2019) for a discussion of the two approaches.

For an MSP problem $(N, L, M, Q, c)$ the associated private game is denoted $C^{P R V}(\cdot, Q)$, or, once again when there is no confusion, $C^{P R V}(\cdot)$. Serving agents in $S$ in the private MSP problem can be solved by the linear program $\gamma^{P R V}(N, L, M, Q, c)$, identical to $\gamma(N, L, M, Q, c)$, except for the additional constraint that $z_{i j}=0$ for all $(i, j) \notin E^{\bar{S}}$.

Given the additional constraints, it is immediate that $C^{P R V}(S) \geq C(S)$ for all $S \subset N$ and $C^{P R V}(N)=C(N)$ and that if $y \in \operatorname{Core}(C)$, then $y \in \operatorname{Core}\left(C^{P R V}\right)$. It is obvious that the opposite is not true. We therefore have the following results.

Lemma 3 For all MSP problems $(N, L, M, Q, c)$, Core $(C) \subseteq \operatorname{Core}\left(C^{P R V}\right)$.

Corollary 2 For all MSP problems $(N, L, M, Q, c)$, Core $\left(C^{P R V}\right)$ is non-empty.

\subsection{Returns to scale}

Another assumption of our model is that we have a linear cost function for each edge. Alternatively, we could have concave or convex cost functions. Assuming concave cost functions on all edges would lead to increasing returns to scale, as edges become cheaper the more they are used. If we take all cost functions to be convex, we get decreasing returns to scale and spreading flow over several paths might be beneficial.

Perhaps surprisingly, if we combine concave cost functions and cooperative gains, we might not be able to find core allocations. Specifically, if the bulk of the gains are generated by small coalitions, the core might be empty. In Trudeau (2009), an example of a (single-period) network flow problem with concave cost functions that has an empty core is provided. The result extends to our multiple period framework.

Though convex cost functions give rise to decreasing returns, this can, however, be partially offset by gains from other factors. In our setup, the ability of an agent to share his unused capacity in a given period is such a gain, and the access to new edges in the private game is another. As the coalition grows, the possibilities to minimize cost increase. 
Suppose first that we have a single period and a public game. In this case, there is no way to compensate the decreasing returns to scale. The core as defined is empty, as coalitions would prefer to act on their own. ${ }^{3}$

A private game, however, offers the possibility to offset the losses caused by the convex cost functions. Quant et al. (2006) consider a (single-period) private network flow game with convex cost functions and show that the core, as defined in this paper, is always non-empty. Although the returns to scale are negative, as a coalition grows, it also gains access to new edges and can now spread its flows to avoid the increasing marginal costs. This is enough to generate a non-empty core.

We can extend the result of Quant et al. (2006) to multiple periods, as long as we keep their assumption of no Steiner nodes. Suppose that for each edge $(i, j)$ we have a convex and increasing cost function $\theta_{i j}\left(k_{i j}\right)$ with $\theta_{i j}(0)=0$. Let $\Theta=\left(\theta_{i j}\right)_{(i, j) \in E}$ and $(N, L, M, Q, \Theta)$ be the resulting network-flow problem with demand over multiple periods. In all our definitions we replace $c$ by $\Theta$. While we no longer have a linear program, we can still express the problem by adapting the objective of problem $\gamma^{P R V}$ to $\min \sum_{(j, k) \in E} \theta_{i j}\left(z_{i j}\right)$.

Theorem 4 If $\Theta$ contains only convex cost functions and $N=L$ then $\operatorname{Core}\left(C^{P R V}\right)$ is non-empty.

Proof. The proof follows Quant et al. (2006).

Let $\lambda$ be a balanced map and $B=\left\{S \in 2^{N} \backslash\{\emptyset\} \mid \lambda_{S}>0\right\}$. For all $S \in B$, let $z^{S}$ and $x^{S}$ be (one of) the solution(s) to $\gamma^{P R V}\left(N, L, M, Q^{S}, \Theta\right)$. Let $z^{*}=\sum_{S \in B} \lambda_{S} z^{S}$ and $x^{*}$ such that $x_{i j t}^{*}=\sum_{S \in B} \lambda_{S} x_{i j t}^{S}$ for all $(i, j) \in E$ and $t \in M$.

We first show that $z^{*}$ and $x^{*}$ satisfy all constraints of problem $\gamma^{P R V}(N, L, M, Q, \Theta)$. To do so, we consider the constraints of problems $\gamma^{P R V}\left(N, L, M, Q^{S}, \Theta\right)$ for all $S \in B$, before summing them up. Constraints $Z_{j k t}$, which only depends on $z^{S}$ and $x^{S}$, are trivially satisfied. We consider constraints $O_{i t} \cdot{ }^{4}$ We have

$$
\begin{aligned}
\sum_{\substack{S \in B \\
i \in S}} \lambda_{S} \sum_{i \in N} x_{i 0 t}^{S} & \geq \sum_{\substack{S \in B \\
i \in S}} \lambda_{S} q_{i t} \\
\sum_{i \in N} \sum_{\substack{S \in B \\
i \in S}} \lambda_{S} x_{i 0 t}^{S} & \geq \sum_{\substack{S \in B \\
i \in S}} \lambda_{S} q_{i t} \\
\sum_{i \in N} x_{i 0 t}^{*} & \geq q_{i t}
\end{aligned}
$$

In the same manner, $z^{*}$ and $x^{*}$ satisfy constraint $I_{i t}$. Thus, $z^{*}$ and $x^{*}$ are potential minimizers for the problem $\gamma^{P R V}(N, L, M, Q, \Theta)$. We show that $\sum_{(j, k) \in E} \theta_{i j}\left(z_{i j}^{*}\right) \leq \sum_{S \in B} \lambda_{S} \sum_{(j, k) \in E} \theta_{i j}\left(z_{i j}^{S}\right)$.

\footnotetext{
${ }^{3}$ The interpretation of the core collapses in this context, as the threat to act on your own to avoid negative externalities is an empty one. It is in fact more natural to reverse the sign of the inequalities in the definition of the core, so that each coalition bears a part of the decreasing returns to scale.

${ }^{4}$ Given that $N=L$, there is no need to use $\bar{q}$ instead of $q$.
} 
We have

$$
\begin{aligned}
\sum_{(j, k) \in E} \theta_{i j}\left(z_{i j}^{*}\right) & =\sum_{(i, j)} \theta_{i j}\left(\sum_{S \in B} \lambda_{S} z_{i j}^{S}\right) \\
& \leq \sum_{(i, j)} \sum_{S \in B} \lambda_{S} \theta_{i j}\left(z_{i j}^{S}\right) \\
& =\sum_{S \in B} \sum_{(i, j)} \lambda_{S} \theta_{i j}\left(z_{i j}^{S}\right) \\
& =\sum_{S \in B} \lambda_{S} C^{P R V}(S)
\end{aligned}
$$

where the inequality follows from the fact that in the private game, only coalitions containing $\{i, j\}$ can use edge $(i, j)$. This implies that $\sum_{\substack{S \in B \\ z_{i j}^{S}>0}} \lambda_{S} \leq 1$ and by the properties of a convex function, the inequality follows.

Then, since $z^{*}$ satisfies all constraints, we have $C^{P R V}(N) \leq \sum_{(j, k) \in E} \theta_{i j}\left(z_{i j}^{*}\right) \leq \sum_{S \in B} \lambda_{S} C^{P R V}(S)$ and thus $C^{P R V}$ is balanced.

Notice that the proof crucially requires that an edge can only be used by coalitions that contain the agents at each end of that edge. Thus, even the presence of a single Steiner node can generate an empty core. The edge between the Steiner node and the source remains public, even in the approach of the private game, and thus vacuity of the core can occur for the same reasons as for the public game.

\subsection{MSP with node ownership}

Another variant of the problem consists in allowing agents to own multiple nodes. There is now a function $\phi: L \rightarrow N \cup\{\emptyset\}$ that tells us who owns each node. If $\phi_{l}=\emptyset$ then node $l$ is a Steiner node. For each $i \in N$, let $\Phi(i)=\{l \in L \mid \phi(l)=i\}$ be the set of nodes owned by $i$. We let $\Phi(T)$ denote the set of nodes owned by agents in $T \subseteq N$. We suppose that $\Phi(i) \neq \emptyset$ for all $i \in N$, i.e. each agent owns at least a node.

Demand is now defined for each owned node: for each $l \in \phi(N), q_{l}=\left(q_{l 1}, \ldots, q_{l m}\right)$ is the demand profile associated to node $l$ and $Q=\left(q_{l}\right)_{l \in \phi(N)}$ is the demand profile.

A multi-period shortest path problem with node ownership is $(N, L, M, \phi, Q, c)$. The cost of supplying agents in $S$ is denoted $C(S, L, \phi, Q)$. To distinguish, the 'classic' MSP problem has a cost $C(S, L, Q)$.

Theorem 5 For any MSP with node ownership $(N, L, M, \phi, Q, c)$, the core is non-empty.

Proof. Fix a multi-period shortest path problem with node ownership $(N, L, M, \phi, Q, c)$. Let $(\Phi(N), L, M, I, Q, c)$ be the corresponding problem in which each owned node has a different owner, i.e. where $I(l)=l$ for all $l \in \Phi(N)$. Clearly, $(\Phi(N), L, M, I, Q, c)$ is a "classic" MSP problem, and 
by our results, its core is non-empty. We use this result to show that the core of $(N, L, M, \phi, Q, c)$ is also non-empty. Let $x \in \mathbb{R}^{\Phi(N)}$ be a core allocation of $(\Phi(N), L, M, I, Q, c)$.

By definition, for all $S \subseteq \Phi(N), x(S) \leq C(S, L, I, Q)$. In particular, it holds for each $\Phi(T)$ for some $T \subseteq N$, for which the constraint becomes

$$
\begin{aligned}
x(\Phi(T)) & \leq C(\Phi(T), L, I, Q) \\
& =C(T, L, \phi, Q)
\end{aligned}
$$

Defining $y(T) \equiv x(\Phi(T))$, we obtain $y(T) \leq C(T, L, \phi, Q)$ and thus $y$ is a core allocation for $(N, L, M, \phi, Q, c)$.

\section{Applications}

An interesting feature of multi-period shortest path problems is that they encompass, partly or fully, a large number of well-studied problems. We describe some of those in this section. The proofs for this section are in the appendix.

The games generated by some of these applications are known to be totally-balanced, and thus by Theorem 2 we have a way to represent them as MSP games. However, that construction is based on the coalitional game itself, post optimization. In this section, we build representations that depend on the parameters of the problem, and thus before the optimization that generates the coalitional game. This will allow to better see the differences and similarities between the various applications considered.

\subsection{Source connection problems}

The most obvious application is to the various source-connection problems. We discuss in this subsection (classic) shortest path problems, airport and irrigation problems, minimum cost spanning tree problems and minimum cost arborescence problems.

\subsubsection{Shortest-path problems}

As discussed in the introduction, MSP problems are extensions of the classic shortest path problems. By setting $m=1$, we recover the full set of (classic) shortest path problems. Note that the public game is not particularly interesting, as each agent paying the cost of his path(s) is the only allocation in the core. More interesting allocations are found in the core of the private game, which include the possibility of subsidies for well-located agents. See Rosenthal (2013) and Bahel and Trudeau (2014). 


\subsubsection{Airport and irrigation problems}

In airport (Thomson (2007)) and irrigation (Aadland and Kolpin (1998)) problems, agents are connected to the source by a fixed tree $\mathcal{T}$, and must share the cost of each segment of that tree. The cost of a coalition $S$ is the cost of all segments needed to connect its members. An airport problem is an irrigation problem in which the tree is a line, in which case the cost of a coalition is equal to the cost of connecting the agent furthest from the source.

An irrigation problem is characterized by a cost vector $\rho$ for each segment on the tree. We extend $\rho$ to a cost matrix as follows, using the notation $p(i)$ to denote the predecessor of $i$ in the tree. If $(i, j)$ is in the tree, then $c_{i j}=\rho_{i j}$. Starting with the agents closest to the source, recursively define $c_{i 0}=c_{p(i) 0}+c_{i p(i)}$. Then, if $i$ is in $j$ 's path to the source, let $c_{j i}=c_{p(j) i}+c_{j p(j)}$. Otherwise, let $c_{j i}=\max \left\{c_{i 0}, c_{j 0}\right\}$.

We complete the representation as an MSP problem by letting $N=L,|M|=|N|$ and $Q=D^{|N|}$. In words, we have the same number of periods as agents, and each agent demands in a different period. It is then obvious that it is optimal to build a capacity of 1 on each edge of tree $\mathcal{T}$, allowing each agent to send one unit of flow along it and yielding $C(N, L, M, Q, c)=\sum_{i \in N} c_{p(i) i}$, just as for irrigation problems.

It is well known that irrigation problems are totally balanced. We recover the result by their representability as an MSP problem.

Lemma 4 All irrigation problems are representable as MSP problems.

\subsubsection{Minimum cost spanning tree and minimum cost arborescence problems}

In a similar fashion, MSP problems also encompass the well-studied minimum cost spanning tree (mcst) problems, in which the cost function on each edge is a fixed cost that has to be paid if the link is used (in any direction), with the cost not depending on the capacity. It is well established that the cores of the public and private versions of that problem are non-empty (Bird (1976)).

We can obtain mcst problems as MSP problems by letting $N=L,|M|=|N|$ and $Q=D^{|N|}$. It now becomes possible to construct a single tree (with capacity 1 ) connecting all agents to the source as they will each use it in a different period. Because all demands are of one unit, the per-unit cost in MSP problems behaves like the fixed cost of mcst problems.

Lemma 5 If the MSP problem $(N, L, M, Q, c)$ is such that $N=L,|N|=|M|, Q=D^{|N|}$ and $c$ is symmetric, then $(N, L, M, Q, c)$ is equivalent to a mcst problem. In addition, all mcst problems can be written as MSP problems.

The second statement in the lemma is obvious: starting from the mcst problem, composed of $N$ and a cost matrix $c$, the equivalent $M S P$ problem is $\left(N, N, N, D^{|N|}, c\right)$. 
Example 2 We revisit Example 1. Remove the Steiner node $s_{1}$ and suppose that to $N$ and $c$ we add $M=\{1, \ldots, 4\}$ and $Q=D^{4}$, i.e. agent $i$ demands 1 unit in period $i$ and none in the other periods. We can then build a minimum cost spanning tree composed of edges (4,2), (2,1), (1,3) and (3,0), for a total cost of 12 . We build a capacity of 1 on each of those edges, and in period $i$, agent $i$ uses the path on that tree from $i$ to the source.

Minimum cost arborescence (mca) problems are extensions of mcst problems where the cost matrix might be asymmetric. The core of both the private and public games are non-empty, and the cooperative games generated by these problems were studied in Dutta and Mishra (2012) and Bahel and Trudeau (2017). The link with MSP problems is the same as for mcst problems, except that we now allow for asymmetric cost functions.

Lemma 6 If the MSP problem $(N, L, M, Q, c)$ is such that $N=L,|N|=|M|, Q=D^{|N|}$, then $(N, L, M, Q, c)$ is equivalent to a mca problem. In addition, all mca problems can be written as MSP problems.

It would be tempting at this point to conclude that public mest and mca problems are totallybalanced, as they can be represented as MSP problems, which are totally-balanced. However, we have counter-examples showing that public mcst problems do not have to be totally-balanced (Norde et al., 2001).

To explain this apparent contradiction, we need to define Steiner tree problems. Steiner tree problems are identical to mcst/mca problems, with the exception that we have public nodes not occupied by any agent. While our framework allows for Steiner nodes, Steiner tree problems can generate games with empty cores (Skorin-Kapov, 1995), meaning that they cannot be represented as MSP problems. We explain the apparent disparity.

Consider a problem with 3 agents (1,2 and 3$)$, and 3 Steiner nodes $\left(s_{1}, s_{2}\right.$ and $\left.s_{3}\right)$. Costs are represented in Figure 2, with drawn edges having a cost of 1 and others having a cost of 10 .

In the Steiner tree problem, any pair of agents can connect at a total cost of 3 (with both connecting to the Steiner node they share a cheap connection to) while the three agents together connect at a total cost of 5 (with one pair connecting as before, and the third agent connecting through another Steiner node). The core of this game is empty.

In the corresponding MSP problem with the same graph, and with agent $i$ demanding one unit in period $i$ and none in other periods, the grand coalition can do better in the following way: build capacities of $1 / 2$ on each of the represented edges, for a total cost of 4.5 and let each agent send half of its flow via one Steiner node and the other half via another. The corresponding game is then balanced.

Thus, we can view Steiner tree problems as an MSP problem with the additional constraint that installed capacities must be 0 or 1 . The idea behind mcst/mca and Steiner tree problems is 


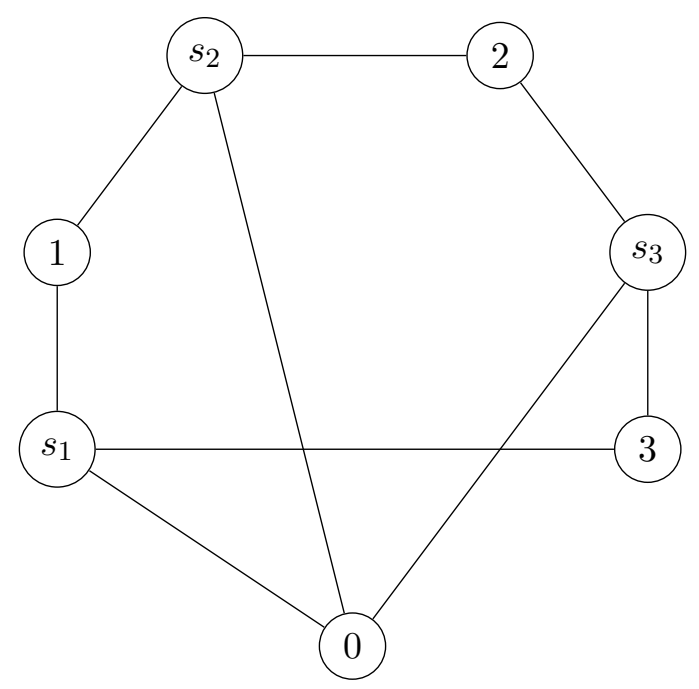

Figure 2: Steiner tree problem

to be connected to the source; for mcst/mca that could be represented by each agents demanding a single unit in a different period, as it was never (uniquely) optimal to send fractions of this unit via different paths. With the presence of Steiner nodes, this is no longer guaranteed, creating a gap between Steiner tree problems and MSP problems.

This is what happens in public mcst/mca problems: We can reinterpret the example above as initially having 6 agents; that game is balanced. But the subgame with agents $\{1,2,3\}$ still allows them to connect through $\left\{s_{1}, s_{2}, s_{3}\right\}$, making that subgame equivalent to the Steiner tree problem of the example. Private mcst/mca problems do not exhibit this problem.

More generally, for total balancedness to extend, we need that the subgames $C_{S}$ remain games of the same type as the original game. This is not necessarily true for source connection problems, as the example shows that a mcst problem can become a Steiner tree problem.

\subsection{Assignment problems}

MSP problems are particularly apt at representing problems in which we match agents together, for instance buyers and sellers. In this section we first explore classic, two-sided assignment problems (Shapley and Shubik (1971)), before examining more general $m$-sided assignment problems. These represent an extension of classic assignment problems in which we add to the number of sides in the market. In the next section we instead extend by keeping the two sides, but complexifying the market in various ways.

\subsubsection{Classic assignment problems}

In classic assignment problems we have two sets of agents and there is a value to match agents from different sets. In an assignment, an agent can be assigned to at most one agent from the other side, 
and the value created is pair-specific. An application is a market for houses. The well-studied model always has a non-empty core. We show in this section that all assignment problems can be written as MSP problems. This result is much less obvious than the previous subsection, as it involves defining $c$ in a precise way to reflect the pair-specific benefits of matching, before reinterpreting the cost game as a savings game.

An assignment problem is a tuple $\left(N^{1}, N^{2}, v\right)$, where $N^{t}$ is the set of agents on side $t$ of the market and $v=\left(v_{i j}\right)_{i \in N^{1}, j \in N^{2}}$ gives the value created by each pair of agents from different sides. We have that $N=\cup_{t} N^{t}$ and for all $S \subset N, S^{t}=S \cap N^{t}$,

An eligible assignment is a set of pairs $a \subset N^{1} \times N^{2}$ such that if $(i, j) \in a$, there is no $k \neq j$ such that $(i, k) \in a$ or $l \neq i$ such that $(l, j) \in a$.

Let $\Omega\left(N^{1}, N^{2}\right)$ be the set of eligible assignments. To find the optimal assignment we need to find $a^{*}$ such that

$$
a^{*} \in \arg \max _{a \in \Omega\left(N^{1}, N^{2}\right)} \sum_{(i, j) \in a} v_{i j} .
$$

Let $V^{A}(S)=\max _{a \in \Omega\left(S^{1}, S^{2}\right)} \sum_{(i, j) \in a} v_{i j}$ for all $S \subseteq N$.

We build an MSP representation based on parameters of the problem, instead of on its corresponding cost function. As can be expected, the way to model assignment problems as MSP problems is to have two periods, with agents demanding in only one of the two periods.

Example 3 We reconsider Example 1. Suppose that to $N \cup\left\{s_{1}\right\}=L$ and $c$ we add $M=\{1,2\}$ and $Q=((1,0),(1,0),(0,1),(0,1))$, i.e. agents 1 and 2 are on one side of the market and 3 and 4 are on the other.

Agents 1 and 3 can join forces by building the path $((1,3),(3,0))$ at a cost of 6 ; a saving of 3 compared to both of them connecting directly to the source. In the same way, agents 1 and 4 would obtain a saving of 4 by building the path $((4,1),(1,0))$, agents 2 and 3 generate a saving of 2 by building the path $((2,3),(3,0))$ and agents 2 and 4 generate a saving of 5 by building a capacity of 1 on $\left(2, s_{1}\right),\left(4, s_{1}\right)$ and $\left(s_{1}, 0\right)$.

Thus, the optimal assignment is to match agent 1 with agent 3 and agent 2 with agent 4 , for total savings of 8 .

We show that the full set of assignment problems can be written as MSP problems in the following way: Let $N=N^{1} \cup N^{2}, L=N,|M|=2$ and $Q$ such that $q_{i}=(1,0)$ if $i \in N^{1}$ and $q_{i}=(0,1)$ otherwise. Let $v^{\max }=\max _{i \in N^{1}, j \in N^{2}} v_{i j}$. Let $c_{i 0}=2 v^{\max }, c_{i j}=c_{j i}=2 v^{\max }-v_{i j}$ if $i \in N^{1}$ and $j \in N^{2}$, and $c_{i j}=c_{j i}=2 v^{\max }$ otherwise.

Lemma 7 All assignment problems $\left(N^{1}, N^{2}, v\right)$ can be written as an MSP problem. 


\subsection{2 $m$-sided assignment problems}

Many extensions of the assignment problem to $m>2$ sides have been proposed, and we focus here on the extensions proposed by Quint (1991) and Atay et al. (2016). Both extensions consider cases in which we have $m$ sides, and value is created by $m$-tuples consisting of one member of each side. While Quint (1991) supposes that these are the only groups of agents creating value, Atay et al. (2016) also allow for subsets to create value. We call the model proposed by Quint (1991) the strict $m$-sided assignment problem and the one by Atay et al. (2016) the generalized $m$-sided assignment problem.

Our set of agents $N$ is now partitioned in $m$ sides, $N^{1}, \ldots, N^{m}$. For $k \in\{2, \ldots, m\}$, let $\mathcal{A}^{k}=\left\{S \subseteq N|| S \mid=k\right.$ and $\left|S^{l}\right| \leq 1$ for $\left.l \in\{1, \ldots, m\}\right\}$ be the set of groups containing $k$ agents from different markets. Let $\mathcal{A}^{2, m}=\bigcup_{k=2}^{m} \mathcal{A}^{k}$.

The strict $m$-sided assignment problem is given by $\left(\left(N^{1}, \ldots, N^{m}\right), w\right)$, with $w \in \mathbb{R}_{+}^{\mathcal{A}^{m}}$ giving the value created by any tuple of $m$ agents from different sides. The generalized $m$-sided assignment problem is given by $\left(\left(N^{1}, \ldots, N^{m}\right), w\right)$, with $w \in \mathbb{R}_{+}^{\mathcal{A}^{2, m}}$.

For the strict $m$-sided assignment problem an eligible assignment is a set of $m$-tuples $a$ such that if $S \in a$, there is no other $S^{\prime}$ in $a$ such that $S^{\prime} \cap S \neq \emptyset$. Let $\Omega\left(N^{1}, \ldots, N^{m}\right)$ be the set of eligible assignments. For the generalized $m$-sided assignment problem an eligible assignment is a set of tuples $a$ of size $m$ or less such that if $S \in a$, there is no other $S^{\prime}$ in $a$ such that $S^{\prime} \cap S \neq \emptyset$. Let $\Omega^{G}\left(N^{1}, \ldots, N^{m}\right)$ be the set of eligible assignments in this case.

To find the optimal assignment for the strict $m$-sided assignment problem we need to find $a^{*}$ such that

$$
a^{*} \in \arg \max _{a \in \Omega\left(N^{1}, \ldots, N^{m}\right)} \sum_{S \in a} w_{S}
$$

while for the generalized $m$-sided assignment problem we are looking for $a^{*}$ such that

$$
a^{*} \in \arg \max _{a \in \Omega^{G}\left(N^{1}, \ldots, N^{m}\right)} \sum_{S \in a} w_{S}
$$

Let $V^{S}(S)=\max _{a \in \Omega\left(S^{1}, \ldots, S^{m}\right)} \sum_{T \in a} w_{T}$ and $V^{G}(S)=\max _{a \in \Omega^{G}\left(S^{1}, \ldots, S^{m}\right)} \sum_{T \in a} w_{T}$ for all $S \subseteq$ N.

Quint (1991) and Atay et al. (2016) report that both the strict and the generalized 3-sided assignment problems can have an empty core, and they offer subsets that are always stable. Stuart Jr (1997) offers a different subset of strict $m$-sided assignment problems that are stable which neither contains nor is contained by the set proposed by Quint (1991). We show that a more general version of this set can be modelled as MSP problems.

We start with the strict $m$-sided assignment problem. Suppose that $S$ is a coalition consisting of one agent from each side. Stuart Jr (1997) shows that the game is stable if we can order the sides $N^{1}, \ldots, N^{m}$ and there exist values $d_{i j}$ for all $\{i, j\} \in N^{r} \times N^{r+1}$ such that for any $S=\left\{i_{1}, \ldots ., i_{m}\right\}$ 
with $S \in \mathcal{A}^{m}$

$$
w_{S}=\sum_{r=1}^{m-1} d_{i_{r} i_{r+1}}
$$

where $i_{r} \in N^{r}$ for all $1 \leq r \leq m$.

A more general set of strict $m$-sided assignment problems can be modelled as MSP problem. Take $m-1$ pairs of sides $N^{t} \times N^{s}$ such that taking one pair out of each would give a connected graph. Let $\mathcal{D}$ denote the set of all values $d_{i j}$ for these $m-1$ pairs of sides, i.e. $d_{i j} \in \mathcal{D}$ if and only if $(i, j) \in N^{t} \times N^{s}$ for one of these $m-1$ pairs of sides $N^{t} \times N^{s}$. We say that a strict $m$-sided assignment problem satisfies the Stuart condition if there exist $m-1$ such pairs of sides such that for all $S \in \mathcal{A}^{m}$ :

$$
w_{S}=\sum_{i, j \in S: d_{i j} \in \mathcal{D}} d_{i j}
$$

We say that a strict $m$-sided assignment problem satisfies the linear Stuart condition if these $m-1$ values form a line.

All strict $m$-sided assignment problems satisfying the Stuart condition can be written as MSP problems in the following way: Let $N=\bigcup_{t} N^{t}, L=N,|M|=m$ and for $i \in N^{s}, q_{i t}=1$ for all $t \neq s$ and $q_{i s}=0$. Let $v^{\max }=\max _{S \in \mathcal{A}^{m}} w_{S}$. Let $c_{i 0}=(m-1) v^{\max }$ for all $i \in N, c_{i j}=c_{j i}=v^{\max }-d_{i j}$ if $i \in N^{t}$ and $j \in N^{s}$ with $t \neq s$, where $d_{i j}=0$ for all $d_{i j} \notin \mathcal{D}$, and let $c_{i j}=v^{\text {max }}$ otherwise.

Theorem 6 Let $\left(\left(N^{1}, N^{2} \ldots, N^{m}\right), w\right)$ be a strict $m$-sided assignment problem which satisfies the Stuart condition. Then $\left(\left(N^{1}, N^{2} \ldots, N^{m}\right), w\right)$ can be written as an MSP problem.

Corollary 3 Every strict $m$-sided assignment problem $\left(\left(N^{1}, \ldots, N^{m}\right), w\right)$ that satisfies the Stuart condition is totally balanced.

Example 4 Consider a strict 3-sided assignment problem with $N^{1}=\{1,2\}, N^{2}=\{3,4\}$ and $N^{3}=\{5\}$. Let $w_{135}=14, w_{145}=11, w_{235}=12$ and $w_{245}=15$. These values satisfy the Stuart condition as we can set $d_{13}=5, d_{14}=4, d_{23}=3, d_{24}=8, d_{35}=9$ and $d_{45}=7$ and then $w_{i j k}=d_{i j}+d_{j k}$, for all $i \in N^{1}, j \in N^{2}$ and $k \in N^{3}$.

We represent this assignment problem as an MSP by using the cost matrix in Figure 3, with $b=15$. In addition, we have $q_{i t}=0$ if $i \in N^{t}$ and $q_{i t}=1$ otherwise.

Note that in this cost matrix $d_{15}=2\left(c_{15}=b-2\right)$ and $d_{25}=3$ rather than 0 . We will use these values in Example 5. For this example, the important thing to note is that, since $(1,5)$ and $(2,5)$ are the two most expensive edges between any two agents from different sides, these edges will not be used by any triplet and hence the MSP problem in this example is equivalent to one in which the cost of these two edges is set to be equal to $b$.

Given the demands, pairs can do no better than having their members connect directly to the source, generating no benefit. Agents belonging to the same side also have no incentives to cooperate. 


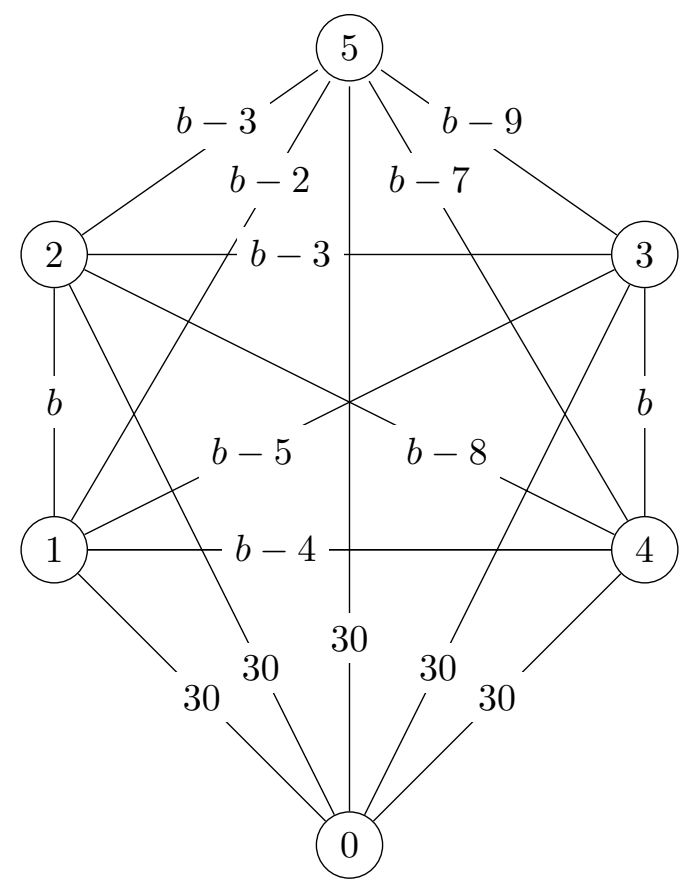

Figure 3: MSP represensation of a strict 3-sided assignment problem.

Sets of 3 agents containing one agent from each side do, however. Take $\{1,3,5\}$. Independently, they each connect directly to the source at a total cost of 90. Together, they can build a capacity of one on $(5,3)$ and $(1,3)$ and two on $(3,0)$, for a total cost of $6+10+2 * 30=76$, and thus a gain of 14. We can verify that for all $i \in N^{1}, j \in N^{2}$ and $k \in N^{3}$, it is optimal for coalition $\{i, j, k\}$ to build a network with one of capacity on $(k, j)$ and $(i, j)$ and two on $(j, 0)$, generating the value in $w$.

The optimal assignment here builds coalition $\{2,4,5\}$ and leaves agents 1 and 3 unassigned, which in our MSP representation implies a direct connection to the source.

Stuart Jr (1997)'s condition for strict assignment problems can be rewritten for generalized $m$-sided assignment problems in the following way. We say that a generalized $m$-sided assignment problem satisfies the linear Stuart condition if we can order the sides $N^{1}, \ldots, N^{m}$ such that for any $S \in \mathcal{A}^{2, m}, w_{S}$ is equal to the sum of the $|S|-1$ highest values of pairs in $S$, and for any $i, j, k \in N$ with $i \in N^{t}, j \in N^{r}, k \in N^{s}, w_{i j} \geq w_{i k}$ if $t<r<s$ or $t>r>s$.

We model these generalized $m$-sided assignment problems in a similar way to how we modelled 2-sided assignment problems as MSP problems. We set $N=\bigcup_{t} N^{t}, L=N,|M|=m$ and $Q$ such that $q_{i t}=1$ if $i \in N^{t}$ and $q_{i s}=0$ otherwise.

Let $v^{\max }=\max _{S \in \mathcal{A}^{m}} w_{S}$. Let $c_{i 0}=2 v^{\max }$ for all $i \in N, c_{i j}=c_{j i}=2 v^{\max }-w_{i j}$ if $i \in N^{t}$ and $j \in N^{s}$, with $t \neq s$ and $c_{i j}=c_{j i}=2 v^{\max }$ otherwise. 
Theorem 7 Let $\left(\left(N^{1}, \ldots, N^{m}\right), w\right)$ be a generalized m-sided assignment problem which satisfies the linear Stuart condition. Then $\left(\left(N^{1}, \ldots, N^{m}\right), w\right)$ can be written as an MSP problem.

Corollary 4 Every generalized $m$-sided assignment problem $\left(\left(N^{1}, \ldots, N^{m}\right), w\right)$ that satisfies the linear Stuart condition is totally balanced.

To illustrate our result, we revisit Example 4.

Example 5 Consider the assignment problem of Example 4. We transform it into a generalized 3-sided assignment problem by defining $w$ as follows. If $|S|=3$, then $w_{S}$ is as in Example 4. For pairs, let $w_{15}=2, w_{25}=3$ and $w_{i j}=d_{i j}$ otherwise.

We represent this problem as an MSP by using the cost matrix in Figure 3, with $b=30$. In addition, we have $q_{i t}=1$ if $i \in N^{t}$ and $q_{i s}=0$ otherwise.

For any coalition $S$ composed of agents from different sides, it is always optimal to build a path of capacity one, starting from the agent in the "last" side and moving to "earlier" sides and then the source, with the sides naturally ranked as 1,2,3. For instance, coalition $\{1,3,5\}$ builds the path $(5,3),(3,1),(1,0)$ at a cost of $21+25+30=76$. Alone, each agent would build a direct link to the source, at a cost of 30 , thus we get a total gain of 14 . Coalition $\{1,5\}$ builds the path $(5,1),(1,0)$ at a cost of $28+30=58$, for a gain of 2 . We can verify that all pairs and triplets containing at most one agent from each side generates the same value as in $w$.

The optimal assignment here builds coalitions $\{1,3,5\}$ and $\{2,4\}$, which generates a value of 22.

\subsection{Market games}

Returning to two sides - buyers and sellers - we show that many ways to model the interactions between buyers and sellers (see Núñez and Rafels (2015) for a review of the vast literature) can be represented as MSP problems.

\subsubsection{Market games with one good}

We extend classic assignment games by relaxing the assumption that agents can only be involved in trades for one unit. Suppose that $N=S \cup B$, where $S$ is a set of sellers and $B$ a set of buyers. Suppose that buyers demand multiple units, with a non-increasing marginal utility, and sellers can produce multiple units, with a non-decreasing marginal cost of production. Then, we can represent the problem as an MSP problem with node ownership.

If a seller has increasing marginal cost of production, it has multiple nodes with demand $(0,1)$. In the same way, if buyers have demand for multiple units of the same good, with decreasing marginal utility, we can represent it with multiple nodes having a demand of $(1,0)$. 
Let $c_{i 0}=c_{j 0} \equiv c_{0}$ for all $i, j \in N$. If $b_{l}$ is the node representing the $l^{t h}$ unit consumed by buyer $b$ and if $s_{k}$ is the node representing the $k^{t h}$ unit produced by a seller $s, c_{b_{l} s_{k}}$ is representative of the value created i.e. such that $c_{0}-c_{b_{l} s_{k}}$ is equal to the surplus created by selling the $k^{\text {th }}$ unit to agent $b$, for whom it is the $l^{t h}$ unit consumed. If it is optimal to "connect" the node of a buyer directly to the source, with no connection to any seller, his demand goes unfulfilled. If it is optimal to "connect" the node of a seller directly to the source, that (potential) supply is not produced. It will always be optimal to first match the highest marginal utilities with the cheapest cost first, which is also why we can't accommodate cases with decreasing marginal cost of production or increasing marginal utility.

Given that agents on the same side of the market have the same demand, they never have any gains to cooperate in the market games as in the corresponding MSP problems.

\subsubsection{Market games with multiple goods}

We can extend the construction from a unique good to multiple goods, as long as the utility and cost are additively-separable over goods.

If these constraints are satisfied, we can build a separate node for each good/unit, i.e. $b_{l k}$ is the node used for the consumption of the $k^{\text {th }}$ unit of good $l$ by buyer $b$. We construct the demand $Q$ in the following way: For each $l \in L$ such that $\phi(l) \in B, q_{l}$ is such that $q_{l t}=0$ for all $t$ except one, i.e. there is an active demand in a single period. In other words, we create an equivalence between the set of periods and the set of goods, and say that node $l$ has a demand for the good $t$ for which the demand is 1 . For each $l \in L$ such that $\phi(l) \in S$, we construct $q_{l}$ such that $q_{l t}=1$ for all $t$ except one. We say that node $l$ has a supply for the good $t$ for which $q_{l t}=0$.

Once again, let $c_{i 0}=c_{j 0} \equiv c_{0}$ for all $i, j \in L . c_{b_{l k} s_{l m}}$ is representative of the value created if buyer $b$ buys its $k^{t h}$ unit of good $l$ from seller $s$, for which it is the $m^{t h}$ such unit produced i.e. such that $c_{0}-c_{b_{l k} s_{l m}}$ is equal to the surplus created by that trade. Note that edges between 'buyer' nodes have a cost of $c_{0}$ since 'buyer' nodes do not create any value together. Thus, even though the demand of some 'buyer' nodes may be compatible, they cannot benefit from connecting to each other. Then, by construction, a 'buyer' node will either be matched with a 'seller' node of the same good, or not matched at all, in which case the demand goes unfulfilled.

Lemma 8 Any market game with multiple goods can be represented as an MSP game, as long as i) marginal utility for each good is non-increasing, ii) marginal cost for each good is non-decreasing, iii) utility and cost are additively-separable over goods.

It is worth noting that the market games described in this section differ from those studied by Shapley and Shubik (1969), which also are equivalent to totally balanced games. Instead of dividing agents into buyers and sellers, they have endowments, with gains coming from mutually 
beneficial trades. While the endowments are not an issue to represent as MSP problem - we can create separate nodes for an agent's endowments and demands - Shapley and Shubik allow utility functions that are not quasi-linear, which we cannot represent in the MSP framework. Thus, while the post-optimization Shapley-Shubik games can be modelled as MSP games through Theorem 2, we cannot represent all pre-optimization problems as MSP problems.

Many variants proposed to our market games (Kaneko (1976), Sotomayor (1992)) incorporate constraints on the number of matchings an agent or a buyer-seller pair can make. These can be added to the MSP problem as follows. Suppose that buyer $i$ cannot consume more than $k$ units (among all goods). We add $k$ additional dummy agents and instead of representing the surplus created by the matching on the edge from the buyer to the seller, we represent it on the path from the buyer to his dummy agent to the seller, with the direct edge presenting no savings for the buyer-seller pair. We now also need to add one more dummy period to $M$ and set the demand of the dummy agents equal to one in each period except for that dummy period, while the buyer and seller nodes have a demand of 1 in that dummy period. This is now equivalent to a strict 3 -sided assignment problem ${ }^{5}$; the buyer and seller node together have a peak demand of two in the dummy period and can thus not create any cost savings. Together with a dummy node, the total demand is two in every period and cost savings become possible.

If we have a market game with multiple goods and constraints on the amount of matchings an agent can make, we need to add two more periods; one 'buyer' period, for which each buyer node and dummy node have a demand of 1 and one 'seller' period, for which each seller node and dummy node have a demand of 1 . Note that this is needed to ensure that two buyer nodes do not create savings together. For market games without constraints we ensured this by setting the cost of an edge between two buyer nodes equal to the cost of an edge to the source. As we now represent the benefits from connecting on the edge towards the dummy node, we need to use the demand vector to ensure that two buyer (or two seller) nodes cannot create any savings together.

Example 6 Consider a market game with two sellers and one buyer. Each seller sells one unit, each of a different good. The buyer has a demand for one unit of each good, but can only buy one unit in total. We represent this as an MSP by using the graph in Figure 4.

We have $N=\{1,2,3,4\}$, with 1 being the buyer, 2 and 3 being the sellers and 4 the dummy agent. We set $\Phi(1)=\left\{b_{11}, b_{21}\right\}, \Phi(2)=\left\{s_{11}\right\}, \Phi(3)=\left\{s_{21}\right\}$ and $\Phi(4)=\left\{d_{1}\right\}$. In addition to that we have $q_{b_{11}}=(1,0,1,0,1), q_{b_{21}}=(0,1,1,0,1), q_{s_{11}}=(0,1,0,1,1), q_{s_{21}}=(1,0,0,1,1)$ and $q_{d_{1}}=(1,1,1,1,0)$. The first period represents the demand/supply of the first good. A buyer $i$ has a demand for good 1 if $q_{i 1}=1$. A seller node $j$ on the contrary supplies this good if his demand in that period is zero, i.e. if $q_{j 1}=0$. Period 2 represents the same but for good 2. The third period is

\footnotetext{
${ }^{5}$ Just as for strict $m$-sided assignment problems, we now also have to increase the cost of an edge to the source to $2 c_{0}$, while keeping the cost of edges between two agents the same, to ensure the cost savings indeed represent the value created by a match between a buyer node and a seller node.
} 
the 'buyer' period, hence $q_{i 3}=1$ if $i$ is a buyer node and $q_{i 3}=0$ if $i$ is seller. For period 4 this is the other way around. The dummy has a demand of one in both of these periods. Lastly, period 5 is our dummy period and $q_{i 5}=0$ only if $i$ is a dummy node.

The cost matrix is as described in Figure 4, where $v_{b_{11}}$ represents the value for the buyer from buying his first unit of good 1 and $\kappa_{s_{11}}$ is the price this seller charges for selling his good. All omitted edges have a cost of $c_{0}$.

Given the demands, no pair can do better than each connecting to the source on its own. Sets of 3 agents can only benefit from cooperation if it consists of 1 buyer, 1 seller and 1 dummy and moreover the buyer node has a demand for the good the seller node supplies. Any other set of 3 agents has a total demand of 3 in one of the periods and can thus not create any cost savings.

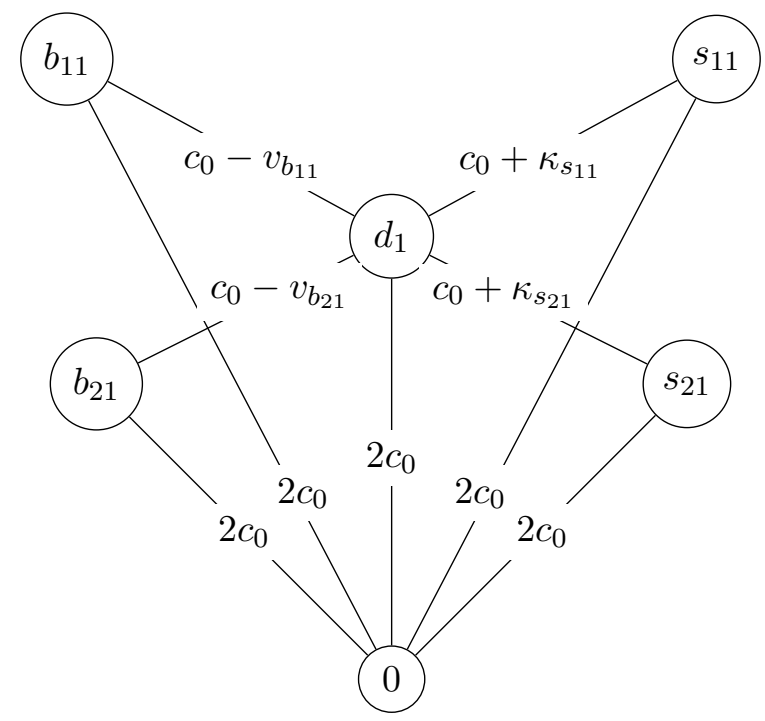

Figure 4: MSP represensation of a market game with constraints and multiple goods.

For constraints on buyer-seller pairs, we proceed in the same manner, creating a dummy agent for each matching a pair can make.

\subsection{Compatibility problems}

The minimum coloring problem is a classic operations research problem in which we need to partition a group into elements that have no conflicts with each other - we can think of scheduling for instance. Conflicts, or incompatibilities, are expressed in a graph composed of undirected edges, that we express as $\{i, j\}$. We have that $i$ and $j$ are incompatible if the undirected edge $\{i, j\}$ belongs to the graph. Okamoto (2008) studies the problem in which we want to minimize the cost of providing services to all agents, with each element of the partition having the same cost. For example we pay $k$ if we need to schedule $k$ different time slots. 
Formally, a minimum coloring problem is $(N, G)$, where $G$ is an undirected graph. For all $S \subset N$, let $G[S]$ be the subgraph induced by $S$. Let $\chi(G)$ be the chromatic number of $G$, i.e. the minimum number of elements in a partition of $N$ so that if $T$ is an element of the partition, $G[T]=\emptyset$. The coalitional cost function associated with the minimum coloring problem $(N, G)$ is $C^{M}(S, G)=\chi(G[S])$.

For a graph $G, S \subseteq N$ is a clique of $G$ if $\{i, j\} \in G$ for all $i, j \in S$. Let $\mathcal{W}(G)$ be the set of cliques. A clique is maximal if there is no other clique that contains it. Let $\overline{\mathcal{W}}(G)$ be the set of maximal cliques. Let $\omega(G)=\max _{S \in \mathcal{W}(G)}|S|$ be the size of the largest clique. Note that $\chi(G) \geq \omega(G)$. We say that a graph $G$ is a weakly perfect graph if $\chi(G)=\omega(G)$. We say that a graph $G$ is a perfect graph if $\chi(G[S])=\omega(G[S])$ for all $S \subseteq N$.

Okamoto (2008) shows that the core of a minimum coloring problem is totally balanced if and only if $G$ is a perfect graph. These can all be represented as MSP problems. For a graph $G$, we build $Q^{G}$ as follows: order cliques in $\overline{\mathcal{W}}(G)=\left\{W_{1}, \ldots, W_{|\overline{\mathcal{W}}(G)|}\right\}$. For all $t=1, \ldots,|\overline{\mathcal{W}}(G)|$ and all $i \in N$, let $q_{i t}^{G}=1$ if $i \in W_{t}$ and $q_{i t}^{G}=0$ otherwise.

Lemma 9 Let $(N, G)$ be a minimum coloring problem, with $G$ a perfect graph. Let $\left(N, N, M, Q^{G}, c\right)$ be such that $m=|\overline{\mathcal{W}}(G)|, c_{i 0}=1$ for all $i \in N$ and $c_{i j}=0$ otherwise. Then, $C^{M}(\cdot, G)=C\left(\cdot, Q^{G}\right)$.

We can provide an additional result: minimum coloring problems for which the graph is weakly perfect still have non-empty cores.

Lemma 10 Let $(N, G)$ be a minimum coloring problem, with $G$ a weakly perfect graph. Then, Core $\left(C^{M}\right)$ is non-empty. ${ }^{6}$

A particular subset of minimum coloring problems is the set of job scheduling problems (Bahel and Trudeau (2019)). In those problems, each agent has a single job that has a starting and finishing time, and must be processed on a machine without interruption from the starting to the finishing time. Evidently, a group of agents is incompatible if their jobs intersect. This gives a lot of structure to the incompatibility graph, which will always be a perfect graph. Thus the core is always non-empty. We can thus represent those problems as MSP problems. To make the representation even closer to the original problem, we can reorder the periods in $M$ such that for any $i \in N$, if $q_{i r}=q_{i t}=1$, then $q_{i s}=1$ for all $r<s<t$. 


\begin{tabular}{|c|c|c|c|c|}
\hline & $N, L$ & $M$ & \multicolumn{2}{|l|}{$Q$} \\
\hline shortest path & $N=L$ & 1 & \multicolumn{2}{|l|}{ any } \\
\hline airport/irrigation & $N=L$ & $|N|$ & \multicolumn{2}{|l|}{$D^{|N|}$} \\
\hline mcst/mca & $N=L$ & $|N|$ & \multicolumn{2}{|l|}{$D^{|N|}$} \\
\hline assignment & $\begin{array}{c}N=L \\
N=N^{1} \cup N^{2}\end{array}$ & 2 & $Q_{i t}=\{$ & $\begin{array}{l}1 \text { if } i \in N^{t} \\
0 \text { otherwise }\end{array}$ \\
\hline strict $m$-sided assign. & $\begin{array}{c}N=L \\
N=\cup_{k=1}^{m} N^{k}\end{array}$ & $m$ & $Q_{i t}=\{$ & $\begin{array}{l}0 \text { if } i \in N^{t} \\
1 \text { otherwise }\end{array}$ \\
\hline gen. $m$-sided assign. & $\begin{array}{c}N=L \\
N=\cup_{k=1}^{m} N^{k}\end{array}$ & $m$ & $Q_{i t}=\{$ & $\begin{array}{c}1 \text { if } i \in N^{t} \\
0 \text { otherwise }\end{array}$ \\
\hline Market/multiple goods & $\begin{array}{c}L: \text { units } \\
N=N^{1} \cup N^{2}\end{array}$ & 2 & $Q_{i t}=\{$ & $\begin{array}{c}1 \text { if } \phi(i) \in N^{t} \\
0 \text { otherwise }\end{array}$ \\
\hline minimum coloring & $N=L$ & $\begin{array}{c}\text { maximal } \\
\text { cliques }\end{array}$ & $Q_{i t}=\{$ & $\begin{array}{c}1 \text { if } i \text { in max. clique } \\
0 \text { otherwise }\end{array}$ \\
\hline
\end{tabular}

\subsection{Relations among the problems}

We have been able to express many problems as MSP problems, with the table below showing the structure of such representations, with a description of how to set up the set of nodes and periods, as well as the demand. The cost matrix, in all cases, is built from the problem at hand. While the shortest path and minimum cost spanning problems come with a cost matrix that we can directly use, other problems require a careful construction, described in the previous subsections. The table allows to find links among the different problems. Some are known, for example that airport and irrigation problems are subset of minimum cost spanning tree problems. Among the new relationships that we can see is the fact that mcst problems are special cases of generalized $m$-sided assignment problems, such that $\left|N^{k}\right|=1$ for all $k \in\{1, \ldots, m\}$. This allows us to conclude that such cases yield a non-empty core. For strict $m$-sided assignment problems, the result is also true, although the result is less direct: Build an MSP as for a generalized assignment problem (demand and cost structure). As long as $c$ satisfies the triangle inequality, it is optimal to connect all agents together in a tree, and the grand coalition has the same value in the generalized and strict versions of the problem. The subcoalitions will generate more values in the generalized version than in the strict version, but any core allocation (in values) for the generalized assignment problem is also a core allocation for the strict assignment problem. We state these results properly.

\footnotetext{
${ }^{6}$ The result can be obtained without MSP representation, by identifying a clique $S$ with the largest size, and building a graph that contains only edges between members of $S$. This graph is obviously perfect, and thus it has core allocations. Since the grand coalition has the same cost of $|S|$ as in the original graph, with all other coalitions having no larger cost than in the original graph, these core allocations are also in the core of the original game.

We present it with the MSP representation to show how it can be used in various contexts to extend stability results.
} 
Theorem 8 Let $(N, w)$ be a generalized or strict m-sided problem, with $N=\cup_{k=1}^{m} N^{k}$. If $\left|N^{k}\right|=1$ for all $k \in\{1, \ldots, m$.$\} the corresponding game has a non-empty core.$

We can also observe a link between generalized assignment problems and minimum coloring problems. To see this, consider the Böhm-Bawerk horse trading game which is a 2-sided assignment problem in which all buyers are symmetrical, and so are all sellers. We can normalize the value created by any pair to 1 . We can model that as a minimum coloring problem, with the groups of buyers and sellers being the only two maximal cliques. The graph is obviously perfect, and the game is totally balanced. We can extend to generalized $m$-sided games, with all agents on a given side being symmetric, any coalition $S$ of agents in different groups creating a value of $|S|-1$. That satisfies the Stuart condition, and the graph is perfect.

In fact, we can view Böhm-Bawerk's game as the intersection of the set of generalized $m$-sided assignment games and of the set of minimum coloring games. This shows the trade-off we are facing when extending 2-sided assignment games. Minimum coloring problems give up the neat structure of agents as belonging to sides, allowing for more complex relationships between agents. However we constrain the gains obtained by a match to be symmetric. The generalized $m$-sided problems instead allow for different matches to create distinct values, but keep the partition of agents in sides. As we have seen, neither of these restrictions are sufficient by itself to guarantee a non-empty core. Böhm-Bawerk's markets, which have both restrictions, are however always stable, even when extended to $m$ sides (Tejada, 2013).

\section{References}

D. Aadland and V. Kolpin. Shared irrigation costs: An empirical and axiomatic analysis. Mathematical Social Sciences, 35(2):203-218, 1998.

A. Atay, F. Llerena, and M. Núñez. Generalized three-sided assignment markets: core consistency and competitive prices. TOP, 24:572-593, 2016.

E. Bahel and C. Trudeau. Stable lexicographic rules for shortest path games. Economics Letters, 125:266-269, 2014.

E. Bahel and C. Trudeau. Minimum incoming cost rules for arborescences. Social Choice and Welfare, 49:287-314, 2017.

E. Bahel and C. Trudeau. Stability and fairness in the job scheduling problem. Games and Economic Behavior, 117:1 - 14, 2019.

C. Bird. On cost allocation for a spanning tree: A game theoretic approach. Networks, 6:335-350, 1976. 
O. Bondareva. Certain applications of the methods of linear programming to the theory of cooperative games. Problemy Kibernetiki (in Russian), 10:119-139, 1963.

B. Dutta and D. Mishra. Minimum cost arborescences. Games and Economic Behavior, 74:120-143, 2012 .

E. Kalai and E. Zemel. Totally balanced games and games of flow. Mathematics of Operations Research, 7(3):476-478, 1982.

M. Kaneko. On the core and competitive equilibria of a market with indivisible goods. Naval Research Logistics Quarterly, 23(2):321-337, 1976.

H. Norde, S. Moretti, and S. Tijs. Minimum cost spanning tree games and population monotonic allocation schemes. European Journal of Operational Research, 154:84-97, 2001.

A. van den Nouweland, S. Tijs, and M. Maschler. Monotonic games are spanning network games. International Journal of Game Theory, 21:419 - 427, 1993.

M. Núñez and C. Rafels. A survey on assignment markets. Journal of Dynamics \& Games, 2: $227-256,2015$.

Y. Okamoto. Fair cost allocations under conflicts a game-theoretic point of view -. Discrete Optimization, 5:1-18, 2008.

M. Quant, P. Borm, and H. Reijnierse. Congestion network problems and related games. European Journal of Operational Research, 172:919-930, 2006.

T. Quint. The core of an m-sided assignment game. Games and Economic Behavior, 3:487 - 503, 1991.

J. Rosenmüller. The theory of games and markets. North Holland, 1981.

E. C. Rosenthal. Shortest path games. European Journal of Operational Research, 224(1):132 $140,2013$.

L. S. Shapley. On balanced sets and cores. Naval Research Logistics Quarterly, 14:453-460, 1967.

L. S. Shapley and M. Shubik. On market games. Journal of Economic Theory, 1(1):9-25, 1969.

L. S. Shapley and M. Shubik. The assignment game i: The core. International Journal of Game Theory, 1:111-130, 1971.

D. Skorin-Kapov. On the core of the minimum cost steiner tree game in networks. Annals of Operations Research, 57:233-249, 1995. 
M. Sotomayor. The Multiple Partners Game. In M. Majumdar, editor, Equilibrium and Dynamics, Palgrave Macmillan Books, chapter 17, pages 322-354. Palgrave Macmillan, 1992.

H. Stuart Jr. The supplier-firm-buyer game and its m-sided generalization. Mathematical Social Sciences, 34(1):21-27, 1997.

O. Tejada. Analysis of the core of multisided Bhm-Bawerk assignment markets. TOP: An Official Journal of the Spanish Society of Statistics and Operations Research, 21:189-205, 2013.

W. Thomson. Cost allocation and airport problems. RCER Working Papers, (537), 2007.

C. Trudeau. Network flow problems and permutationally concave games. Mathematical Social Sciences, 58:121 - 131, 2009.

C. Trudeau and J. Vidal-Puga. The Shapley value in minimum cost spanning tree problems, in: E. Algaba, V. Fragnelli, J. Sanchez-Soriano (eds), Game Theory: The Shapley value. Taylor and Francis Group, 2019.

\section{A Proofs}

\section{A.1 Lemma 7}

Take a generalized $m$-sided assignment problem with the property that for any coalition $S$ with at most one agent from each group, $w_{S}$ is equal to the sum of the $|S|-1$ pairs in $S$ that create the most value and moreover these $|S|-1$ pairs create a connected component. We first show that for such generalized $m$-sided assignment problems and all 2-sided assignment problems the MSP we constructed based on it is such that $\sum_{e \in E} z_{e}^{*}=|N|$ for any optimal graph $z^{*}$.

Recall our construction of an MSP for 2 -sided and generalized $m$-sided assignment problems is as follows: Given an assignment problem $\left(\left(N^{1}, \ldots, N^{m}\right), w\right)$, let $N=\bigcup_{t} N^{t}, L=N,|M|=m$ and $Q$ such that $q_{i t}=1$ if $i \in N^{t}$ and $q_{i t}=0$ otherwise. Let $v^{\max }=\max _{S \in \mathcal{A}^{m}} w_{S}$. Let $c_{i 0}=2 v^{\max }$, $c_{i j}=c_{j i}=2 v^{\max }-w_{i j}$ if $i \in N^{t}$ and $j \in N^{s}$, with $t \neq s$ and $c_{i j}=c_{j i}=2 v^{\text {max }}$ otherwise.

Lemma A.1 Let $\left(\left(N^{1}, \ldots, N^{t}\right), w\right)$ be a 2-sided assignment problem or a generalized $m$-sided assignment problem such that for any coalition $S$ with at most one agent from each group, $w_{S}$ is equal to the sum of the $|S|-1$ pairs in $S$ that create the most value and moreover these $|S|-1$ pairs create a connected component. Then, in the MSP as defined above, for any optimal network $z^{*}$ we have $\Sigma_{e \in E} z_{e}^{*}=|N|$, i.e. the total capacity is equal to the amount of agents.

Proof. It is easy to see that our cost matrix satisfies the triangle inequality: all edges have a cost of at least $v^{\max }$, and thus a path of 2 edges costs at least $2 v^{\text {max }}$. This means that the cost of a path of 2 edges is at least as large as the cost of a direct connection, which has a cost of at most $2 v^{\max }$. 
Furthermore, since every $i \in N$ has a demand of 1 in one of the periods, the sum of capacities on the outgoing edges from $i$ must be at least 1 . Hence the total sum of capacities on any feasible network must be at least $|N|$. We can furthermore assume that the capacities on all edges are fully used, as otherwise the network would not be optimal.

Now consider a network $z$ with $\sum_{e \in E} z_{e}>|N|$. We will show that such a network is not strictly optimal. As $\sum_{e \in E} z_{e}>|N|$, there must exist at least one node $j$ with outgoing capacity of more than 1 in $z$. As $j$ only needs a total capacity of 1 for its own demand, this means we can find a set of agents $S$ that sends (part of) its flow via $j$ in $z$, such that redirecting this flow would allow us to reduce the amount of capacity on the outgoing edges from $j$. More specifically, we can then find such an $S$ consisting of agents from different sides, and an amount $\alpha$, such that reducing the amount that this coalition $S$ sends via $j$ by $\alpha$ would render an amount of $\alpha$ of the outgoing capacity from $j$ unused (as well as an amount of $\alpha$ on all of the ingoing edges from $S$ into $j$ over which this flow is sent). For sake of simplicity we will moreover assume that the total amount of unused capacity is on one outgoing edge $(j, l)$ only. As we can always pick $\alpha$ small enough for this to be true, this assumption is w.l.g.

We will now compare the cost of $z$ with the cost of an alternative network where the flow of $S$ is redirected and the capacities on the corresponding edges are reduced/increased accordingly. We abuse notation slightly by writing $w_{i j}$ even when nodes $i$ and $j$ may be from the same side or one of them may be the source node, with the understanding that in these cases the cost of the corresponding edge is $2 v^{\max }$, i.e. equal to assuming $w_{i j}=0$.

Let $\bar{S}=\left\{i \in S \mid z_{i j}>0\right\}$ denote the set of agents in $S$ that send flow directly to $j$ in $z$. I.e. when redirecting the flow of $S$, the edges which capacities we will be able to reduce aside from $(j, l)$ are all $(i, j)$ for $i \in \bar{S}$. First, suppose there is an $i \in \bar{S}$ that is from the same side as $j$. As by construction it then holds that $c_{i j}=2 v^{\max }=c_{i 0}$, setting $z_{i j}=0$ and adding a capacity of $z_{i j}$ to $z_{i 0}$ would give a feasible network with exactly the same cost. Hence, we can assume all $i \in \bar{S}$ to be from a different side than $j$ and $|\bar{S}| \leq m-1$.

We now redirect all flow from $S$ over $j$ to edges directly to the source, i.e. we reduce the capacity on all $(i, j)$ for $i \in \bar{S}$ and $(j, l)$ by $\alpha$ and increase the capacity on $(i, 0)$ for all $i \in \bar{S}$ with $\alpha$. This gives a change in cost of:

$$
\begin{array}{r}
\alpha\left(|\bar{S}| 2 v^{\max }-\left(|\bar{S}| 2 v^{\max }-\Sigma_{i \in \bar{S}} w_{i j}\right)-\left(2 v^{\max }-w_{j l}\right)\right)= \\
\alpha\left(\Sigma_{i \in \bar{S}} w_{i j}+w_{j l}-2 v^{\max }\right) \leq 0
\end{array}
$$

Where the inequality follows from the fact that $w_{j l} \leq v^{\max }$ and as $v^{\max }=\max _{S \in \mathcal{A}^{m}} w_{S}$ and $\bar{S}$ contains at most one agent from each side, $\Sigma_{i \in \bar{S}} w_{i j} \leq v^{\max }$ as well.

We are now ready to prove Lemma 7 . 
Proof. As $c$ satisfies the triangle inequality, we have a limited number of paths to consider. Every singleton $\{i\}$ connects through the path $((i, 0))$ at a cost of $2 v^{\max }$, and thus $C(\{i\})=2 v^{\max }$.

Now consider pairs $\{i, j\}$. If they belong to the same group $N^{1}$ or $N^{2}$, then the best they can do is both connect directly to the source, and $C(\{i, j\})=4 v^{\max }$ and $\left.V(\{i, j\})\right)=0$. If they don't belong to the same group, then they can share (part of) the same path. Since they are completely symmetric, we connect $j$ to $i$ and $i$ to the source, at a cost of $4 v^{\max }-v_{i j}$. Thus, $C(\{i, j\})=4 v^{\max }-v_{i j}$ and $V(\{i, j\}))=4 v^{\max }-\left(4 v^{\max }-v_{i j}\right)=v_{i j}$.

Now take an $|S|>2$. From Lemma A.1 we know that in an optimal network, the total amount of capacity for each agent on its outgoing edges must be exactly 1 and the total capacity of the network must thus be exactly $|N|$. This implies that for every agent $i$ his demand is sent either directly to the source or via an agent $j$ from the other side, with whom he shares the capacity on $(j, 0)$, or a mix of these two. We can then for any optimal network $z^{*}$ find a collection of balancing weights $\lambda_{S}$ over $\left\{S \mid S=\{i, j\}, i \in N^{1}, j \in N^{2}\right.$ or $\left.|S|=1\right\}$ and a set of networks $z^{S}$ such that $z^{*}=\sum \lambda_{S} z^{S}$. Particularly, $z^{*}$ will be the weighted sum of the networks $z^{S}$ where for any $S$ such that $|S|=1, z_{i 0}^{S}=1$ and $z_{e}^{S}=0$ otherwise and for $S=\{i, j\}$ either $z_{j i}^{S}=z_{i 0}^{S}=1$ or $z_{i j}^{S}=z_{j 0}^{S}=1$ and $z_{e}^{S}=0$ otherwise.

Since assignment games are known to be balanced, we know that $V^{A}(N) \geq \sum_{(i, j) \in a} \lambda_{\{i, j\}} v_{i j}$ for all sets of balancing weights $\lambda$. As we have shown that $V(\{i, j\})=V^{A}(\{i, j\})=v_{i j}$ for all pairs and that in our MSP an optimal network must correspond to the $\lambda$-weighted sum over networks for pairs and singletons, it then follows that our MSP is indeed equal to the original assignment problem.

\section{A.2 Theorem 6}

Before we show that these MSP problems indeed correspond to the strict $m$-sided assignment problem it is based on, we first show specifically for $S \in \mathcal{A}^{m}$ that $V(S)=V^{S}(S)$.

Lemma A.2 Let $\left(\left(N^{1}, N^{2} \ldots, N^{m}\right), w\right)$ be a strict m-sided assignment problem satisfying the Stuart condition and set $d_{i j}=0$ for all $d_{i j} \notin \mathcal{D}$. Set $N=\bigcup_{t} N^{t}, L=N,|M|=m$ and for $i \in N^{s}$, $q_{i t}=1$ for all $t \neq s$ and $q_{i s}=0$. Let $v^{\max }=\max _{S \in \mathcal{A}^{m}} w_{S}$. Let $c_{i 0}=(m-1) v^{\text {max }}$ for all $i \in N$, $c_{i j}=c_{j i}=v^{\max }-d_{i j}$ if $i \in N^{t}$ and $j \in N^{s}$ with $t \neq s$ and let $c_{i j}=v^{\text {max }}$ otherwise.

Then $V(S)=V^{S}(S)$ for all $S \in \mathcal{A}^{m}$.

Proof. First, note that $c$ satisfies the triangle inequality and we can therefore ignore any edges to nodes outside of $S$. For $S$ it holds that $\sum_{i \in S} q_{i t}=m-1$ for any $t \in M$, i.e. the agents in $S$ could share a total capacity of $m-1$ on edges to the source. Building a network with capacity 1 on either $(i, j)$ or $(j, i)$ for each $d_{i j} \in \mathcal{D}_{S}$, where $\mathcal{D}_{S}$ is the restriction of $\mathcal{D}$ to $S$, and a total capacity of $m-1$ on the edges between the agents in $S$ and the source, has a total cost of $(m-1)(m-1) v^{\max }+(m-1) v^{\max }-\sum_{d_{i j} \in \mathcal{D}_{S}} d_{i j}$. As, by assumption, for all other pairs $i, j \in S$, 
$d_{i j}=0$ it is easy to see that it is not possible to build a cheaper network for $S$. It remains to show that there exists such a network that is indeed feasible for $S$.

The edges $(i, j)$ for $d_{i j} \in \mathcal{D}_{S}$ form a connected component and more specifically a tree. Pick an $i \in S$ which is not a leaf node in this tree. For any $j \in S$ that is a neighbor of $i$ in the tree, we set $z_{j i}=1$ and $z_{i j}=0$. Next, for any node $k \in S$ that is a neighbor of $j$ in the tree we set $z_{k j}=1$ and $z_{j k}=0$. We repeat this process until we have covered all $k \in S$. We now have a directed tree consisting of edges corresponding to the values in $\mathcal{D}_{S}$, with $i$ being the root node of the tree.

What remains is to pick $(m-1)$ edges connecting $S$ to the source. We do this as follows: For any $i \in S$, set $z_{i 0}=\left|\left\{j \in S \mid z_{j i}=1\right\}\right|$, i.e. the capacity on the edge from $i$ to the source is equal to the amount of ingoing edges for $i$ with a capacity of 1 . Since $\left|\mathcal{D}_{S}\right|=m-1$, the total capacity on edges between $S$ and the source is now $m-1$. We will show that this network is indeed feasible for $S$.

First consider a node $k$ which has $x>0$ ingoing edges $(j, k)$ with $z_{j k}=1$, and all of these neighbor nodes $j$ are leaf nodes. Each of these leaf nodes, by construction, has exactly 1 outgoing edge with capacity 1 and thus must send all of his demand over this edge $(j, k)$. As all $j \in S$ are from different sides, each of these agents has his demand of 0 in a different period. Hence, at node $k$ we now have an accumulated demand of $x+1$ ( $k$ 's neighbors plus $k$ 's own demand) in all but $x+1$ of the periods and an accumulated demand of $x$ in the other periods. By construction, $z_{k 0}=x$ and thus after using this capacity fully, we are left with a demand of 1 in each of the periods that corresponds to neither $k$ 's side, nor the side of any of $k$ 's leaf node neighbors. This remaining demand we send over $k$ 's outgoing edge to his neighbor in the direction of the central node $i$.

Repeating this at every node, we can thus see that at each node the demand that is left after sending the maximum possible to the source, is a vector consisting of 1's and 0's and after each node that is not a leaf node, we have at least one fewer period for which the remainder demand is 1 . It then follows that if $i$ has $x^{\prime}$ ingoing edges, the demand that is sent to $i$ from his direct neighbors adds up to $x^{\prime}-1$ for all periods except for the period in which $i$ 's demand is 0 , where it adds up to $x^{\prime}$. The total accumulated demand at $i$ is thus equal to $x^{\prime}$ for every period. As by construction $z_{i 0}=x^{\prime}$, the network is thus feasible.

We are now ready to prove Theorem 6 .

Proof. As each agent has a demand of 0 in one of the periods and 1 in all other periods a coalition can only reduce the total capacity on edges towards the source, compared to acting individually, if it contains at least 1 agent from each side. It thus follows that for any $S$ such that $S \cap N^{t}=\emptyset$ for some $t, V(S)=V^{S}(S)=0$. For all $S \in \mathcal{A}^{m}$ we know from Lemma A.2 that $V(S)=V^{S}(S)$. It thus remains to show that this also holds for coalitions $|S|>m$ with at least one agent from each side. 
As $c$ satisfies the triangle inequality, it is always cheaper to connect directly than via an indirect path, unless this indirect connection allows for the sharing of capacity. Hence, if $z_{i j}>0$ for some edge $(i, j)$, it must be the case that $i$ and $j$ are part of some $m$-tuple $S \in \mathcal{A}^{m}$ in order to benefit from this detour. We can thus conclude that any optimal network for $N$ must be a weighted sum of optimal subnetworks for $S \in \mathcal{A}^{m}$ and singletons connecting directly to the source by themselves.

Next, we can assume for any $(i, j)$ such that $z_{i j}>0$ that $d_{i j} \in \mathcal{D}$. To see this, note that for any $i$ and any $m$-tuple $S \ni i$ there is a $j \in S \backslash\{i\}$ such that $d_{i j} \in \mathcal{D}_{S}$ and $c_{i j} \leq c_{i k}$ for any $d_{i k} \notin \mathcal{D}_{S}$. Recall that $\mathcal{D}$ contains $d_{i j}$ for $(i, j) \in N^{t} \times N^{s}$ for exactly $m-1$ pairs $N^{t} \times N^{s}$. This implies that we can see our problem of finding an optimal network as a problem of finding the optimal way to connect agents in $N^{t}$ with agents in $N^{s}$ for any $N^{t} \times N^{s} \subset \mathcal{D}$, with the small difference that if the smallest side consists of $x$ agents, we connect exactly $x$ of the pairs in $N^{t} \times N^{s}$ even if both $\left|N^{t}\right|>x$ and $\left|N^{s}\right|>x$. Thus, our problem essentially corresponds to solving $m-12$-sided assignment problems. As we know that under our MSP construction for 2-sided assignment games it was indeed optimal to connect the agents in pairs, rather than building a graph that was a weighted average, it is easy to to see that also in our MSP problem here, it is not strictly optimal to build a network that is a weighted average. Hence we may assume that in the optimal network for our MSP, each agent $i$ has at most one edge $(i, j)$ for $j \neq 0$ such that $z_{i j}=1$ and for all other edges from $i$ to any $k \in N \backslash\{j\}, z_{i k}=0$.

We can thus conclude that there is an optimal graph which corresponds to $x$ disjoint subgraphs each corresponding to an $S \in \mathcal{A}^{m}$ and possibly some agents connecting individually to the source. Hence our MSP is equal to the original strict assignment problem.

\section{A.3 Theorem 7}

Proof. Let $(N, M, Q, c)$ be an MSP problem as defined in Section 4.2.2 for a generalized $m$ sided assignment problem. We will show that it corresponds to the original generalized $m$-sided assignment problem $\left(\left(N^{1}, \ldots, N^{m}\right), w\right)$.

It follows from Lemma A.1 that the flow of an agent $i$ never goes via an agent from the same side as $i$. In other words, it either goes directly to the source, or via a path consisting of some agents from a different side from $i$ (which themselves are not from the same side either). Moreover, in the second case $i$ shares the capacity on the edges he uses with the agents he sends his flow via. This means that when examining if an MSP problem indeed corresponds to a generalized $m$-sided assignment problem we only need to consider a limited number of possible networks.

From this it follows that for any optimal network $z^{*}$ we can find a collection of balancing weights $\lambda_{S}$ over $\left\{S \mid S \in \mathcal{A}^{2, m}\right.$ or $\left.|S|=1\right\}$ such that $z^{*}$ is equal to the $\lambda$-weighted sum of networks $z^{S}$ for $S \in \mathcal{A}^{2, m}$ and singletons. It turns out that for $m$-sided assignment problems that satisfy the linear Stuart condition, we can make additional assumptions on the networks $z^{S}$ for $S \in \mathcal{A}^{2, m}$. 
Due to Lemma A.1 we can assume for the singletons that the network $z^{\{i\}}$ has capacity of 1 on $(i, 0)$ and 0 otherwise. By the same result, combined with the symmetry of $c$ and the fact that $c_{i 0}=2 v^{\max }$ for all $i \in N$, we may assume that $z^{\{i, j\}}$, for $i \in N^{s}, j \in N^{t}, t>s$, has a capacity of 1 on $(j, i)$ and $(i, 0)$ and 0 otherwise. In general, for any $S \in \mathcal{A}^{2, m}$ and $i, j, k \in S$ with $i \in N^{r}, j \in N^{s}, k \in N^{t}, t>s>r$ we can assume $z^{S}$ to have a capacity of 0 on $(k, i)$, since $w_{k i} \leq w_{k j}, w_{j i}$ and thus $c_{k i} \geq c_{k j}, c_{j i}$. As moreover $c_{i 0} \geq c_{i j}$ for all $i \in N$ and $j \neq i$ we may in general assume that for any coalition the flow goes from the agent belonging to the side with the highest number in our order to the agent from the side with the lowest number, via all other agents in the coalition in order of decreasing side number. It is easy to see from this that $V(S)=V^{G}(S)$ for all $\left\{S \mid S \in \mathcal{A}^{2, m}\right.$ or $\left.|S|=1\right\}$.

Now let $z$ be a network corresponding to a balanced collection of weights on $\left\{S \mid S \in \mathcal{A}^{2, m}\right.$ or $|S|=$ 1 ) such that there is a $0<\lambda^{S}<1$. We will show that this network cannot be strictly cheaper than one which corresponds to a balanced collection of weights on $\left\{S \mid S \in \mathcal{A}^{2, m}\right.$ or $\left.|S|=1\right\}$ in which all weights are either 0 or 1 , i.e. one that corresponds to a feasible assignment.

Note that we can assume there to be an edge $e$ such that $0<z_{e}<1$, since if $z_{e} \in\{0,1\}$ for all edges $e \in E$, we can always find a balanced collection of weights on $\left\{S \mid S \in \mathcal{A}^{2, m}\right.$ or $\left.|S|=1\right\}$ in which all weights are either 0 or 1 and we would be done. We will show that, for any feasible network $z$ with some $0<z_{e}<1$, we can construct a weakly cheaper network with at least one fewer edge $e$ for which $0<z_{e}<1$.

Since we may assume that all $i \in N^{1}$ send their flow directly to the source, any edge $e$ such that $0<z_{e}<1$ is either between two agents from different sides, or between $j \in N \backslash N^{1}$ and the source. By Lemma A.1 we furthermore know that we can assume $\sum_{i \in N_{0}} z_{j i}=1$ for all $i \in N$.

Next, since for $S \in \mathcal{A}^{2, m}$ we assumed that the path used is a line, going from the agent in the side with the highest number towards the agent in the side with the lowest number, it follows that for every $\left\{S \mid S \in \mathcal{A}^{2, m}\right.$ or $\left.|S|=1\right\}$ and for every $i \in S$, at most one of the ingoing edges for $i$ has a capacity of 1 in $z^{S}$, and all others have a capacity of 0 . As $\lambda$ is a balanced map, it then follows that $\sum_{j \in N} z_{j i} \leq 1$ for all $i \in N$ and moreover that for any side $N^{t}$ and any edge $(j, i)$, the sum of the flows from agents in $N^{t}$ going via $(j, i)$ is at most $z_{j i}$.

We now construct two graphs, $z^{1}$ and $z^{2}$, such that $z$ is the weighted average of $z^{1}$ and $z^{2}$. Let $\left(j_{1}, i_{1}\right)$ be the edge with the smallest non-zero capacity in $z$ and let $\left(j_{1}, i_{2}\right)$ be a different edge such that $0<z_{j_{1} i_{2}}<1$. We set $z_{j_{1} i_{1}}^{\prime}=0, z_{j_{1} i_{2}}^{\prime}=z_{j_{1} i_{2}}+z_{j_{1} i_{1}}$ and $z^{\prime}=z$ otherwise. Next we check if $\sum_{j \in N} z_{j i_{2}}^{\prime} \leq 1$, i.e. if $i_{2}$ does not have more capacity than 1 on his ingoing edges after this change. If $\sum_{j \in N} z_{j i_{2}}^{\prime} \leq 1$ holds or if $i_{2}$ is the source node, we are done. Otherwise, pick a new edge $\left(j_{2}, i_{2}\right) \neq\left(j_{1}, i_{2}\right)$ such that $0<z_{j_{2} i_{2}}<1$. Note that such an edge must exist, since $\sum_{j \in N} z_{j i} \leq 1$ for all $i \in N$. We repeat the same process for $j_{2}$, moving again a capacity of $z_{j_{1} i_{1}}$. I.e. we pick an edge $\left(j_{2}, i_{3}\right) \neq\left(j_{2}, i_{2}\right)$ such that $0<z_{j_{2} i_{3}}<1$ and set $z_{j_{2} i_{2}}^{\prime \prime}=z_{j_{2} i_{2}}-z_{j_{1} i_{1}}, z_{j_{2} i_{3}}^{\prime \prime}=z_{j_{2} i_{3}}+z_{j_{1} i_{1}}$ and $z^{\prime \prime}=z^{\prime}$ otherwise. 
We repeat this process until we find a cycle or chain such that moving a capacity of $z_{j_{1} i_{1}}$ in either direction gives a new network $\tilde{z}$ in which $\sum_{j \in N} \tilde{z}_{j i} \leq 1$ for all $i \in N$. Note that in the case of a chain, and if $\sum_{j \in N} z_{j i_{1}}>1-z_{j_{1} i_{1}}$, we will have to repeat the same process in the opposite direction, starting with adding $z_{j_{1} i_{1}}$ to edge $\left(j_{1}, i_{1}\right)$ and subtracting it from edge $\left(j_{1}, i_{2}\right)$, in order to ensure we have a chain such that moving a capacity of $z_{j_{1} i_{1}}$ in both directions will yield a network in which $\sum_{j \in N} \tilde{z}_{j i} \leq 1$ for all $i \in N$. Since we have a finite amount of edges and the condition $\sum_{j \in N} z_{j i} \leq 1$ is satisfied for $z$, it is always possible to find such a chain or cycle.

Now let $z^{1}$ be the network created when we move a capacity of $z_{j_{1} i_{1}}$ in the initial direction, i.e. $z_{j_{1} i_{1}}^{1}=0$. In the other direction we might be able to move more. Let $z_{j^{\prime} i^{\prime}}$ be the largest amount of capacity we can move in this direction and denote $z^{2}$ the resulting network (thus $z_{j^{\prime} i^{\prime}}^{2}=0$ ). Note that both $z^{1}$ and $z^{2}$ are still feasible networks as by construction $\sum_{j \in N} z_{j i}^{x} \leq 1$ for both $z^{1}$ and $z^{2}$ and at every step we keep $\sum_{i \in N} z_{j i}^{\prime}=1$ for all $j \in N$. Moreover, as we assumed that the flow of every coalition goes in the same direction, we can be sure that both $z^{1}$ and $z^{2}$ are still such that for any side $N^{t}$ and any edge $(j, i)$, the sum of the flows from agents in $N^{t}$ going via $(j, i)$ is at most 1 .

We show that $z=\frac{z_{j^{\prime} i^{\prime}}}{z_{j_{1} i_{1}}+z_{j^{\prime} i^{\prime}}} z^{1}+\frac{z_{j_{1} i_{1}}}{z_{j_{1} i_{1}}+z_{j^{\prime} i^{\prime}}} z^{2}$. Note that for any edge $k l$ that is not part of the cycle/chain $z_{k l}=z_{k l}^{1}=z_{k l}^{2}$. Now consider an edge $k l$ such that $z_{k l}^{2}=z_{k l}-z_{j^{\prime} i^{\prime}}<z_{k l}<z_{k l}+z_{j_{1} i_{1}}=$ $z_{k l}^{1}$. We then have that:

$$
\begin{aligned}
\frac{z_{j^{\prime} i^{\prime}}}{z_{j_{1} i_{1}}+z_{j^{\prime} i^{\prime}}} z_{k l}^{1}+\frac{z_{j_{1} i_{1}}}{z_{j_{1} i_{1}}+z_{j^{\prime} i^{\prime}}} z_{k l}^{2} & = \\
\frac{z_{j^{\prime} i^{\prime}}}{z_{j_{1} i_{1}}+z_{j^{\prime} i^{\prime}}}\left(z_{k l}+z_{j_{1} i_{1}}\right)+\frac{z_{j_{1}}}{z_{j_{1} i_{1}}+z_{j^{\prime} i^{\prime}}}\left(z_{k l}-z_{j^{\prime} i^{\prime}}\right) & = \\
z_{k l}+\frac{z_{j^{\prime} i^{\prime}}}{z_{j_{1} i_{1}}+z_{j^{\prime} i^{\prime}}} z_{j_{1} i_{1}}-\frac{z_{j_{1} i_{1}}}{z_{j_{1} i_{1}}+z_{j^{\prime} i^{\prime}}} z_{j^{\prime} i^{\prime}} & =z_{k l}
\end{aligned}
$$

It is easy to see that the same holds for any edge $k l$ such that $z_{k l}^{2}=z_{k l}+z_{j^{\prime} i^{\prime}}>z_{k l}>z_{k l}-z_{j_{1} i_{1}}=$ $z_{k l}^{1}$. Hence, $z$ is indeed a weighted average of $z^{1}$ and $z^{2}$. By definition of a weighted average, either $z^{1}$ or $z^{2}$ must be weakly cheaper than $z$. Hence we have constructed a feasible network that is weakly cheaper than $z$ and which has at least one fewer edge $e$ such that $0<z_{e}<1$. We can thus conclude that a network corresponding to a balanced collection of weights such that all weights are either 0 or 1 is weakly better than one for which this is not the case.

As $V(S)=V^{G}(S)$ for all $\left\{S \mid S \in \mathcal{A}^{2,3}\right.$ or $\left.|S|=1\right\}$, it then follows that $V(S)=V^{G}(S)$ for all $S \subseteq N$

\section{A.4 Lemma 9}

Proof. First, notice that by definition of $c$, we have that for any network $z, \sum_{e \in E} z_{e} c_{e}=\sum_{i \in N} z_{i 0}$, i.e. the cost is the sum of the capacities to the source. 
Fix $S \subseteq N$. Let $\overline{\mathcal{W}}^{+}(G)=\{T \in \overline{\mathcal{W}}(G)|| T \mid=\omega(G)\}$ and let $G^{S+}$ be such that $\{i, j\} \in G^{S+}$ if and only if $i, j \in R \in \overline{\mathcal{W}}^{+}(G[S])$. For any $G^{\prime} \subset G$, we construct $\mathrm{Q}^{\mathrm{G}^{\prime}}$ from $Q^{G}$ as follows: for all $t=1, \ldots,|\overline{\mathcal{W}}(G)|$, if there exists a $T \in \overline{\mathcal{W}}\left(G^{\prime}\right)$ such that $T \subseteq W_{t}$, we set $\mathrm{q}_{\mathrm{it}}^{\mathrm{G}^{\prime}}=q_{i t}^{G}$ for all $i \in T$. Otherwise $q_{i t}^{G^{\prime}}=0$.

We consider the problem $\left(N, M, \mathrm{Q}^{\mathrm{G}^{\mathrm{S}}}, c\right)$ and in particular $C\left(S, \mathrm{Q}^{\mathrm{G}^{\mathrm{S}}}\right)$. Take any $R \in \overline{\mathcal{W}}^{+}(G[S])$. Then, $\{i, j\} \in G^{S+}$ for all $i, j \in R$ and by construction of $\mathrm{Q}^{\mathrm{G}^{\mathrm{S}}}$, there exists $t \in M$ such that $\mathrm{q}_{\mathrm{it}}^{\mathrm{G}^{\mathrm{S}+}}=1$ for all $i \in R$. To accommodate this demand in period $t$, we will need (at least) $|R|=\omega(G[S])$ units of capacity to the source, i.e. we need that $\sum_{i \in N} z_{i 0} \geq|R|=\omega(G[S])$. Thus, we have $C\left(S, \mathrm{Q}^{\mathrm{G}^{\mathrm{S}}}\right) \geq \omega(G[S])=\chi(G[S])$, where the last equality comes from $G$ being a perfect graph.

Next, observe that $\left(Q^{G}\right)^{S} \geq \mathrm{Q}^{\mathrm{G}[\mathrm{S}]} \geq \mathrm{Q}^{\mathrm{G}^{\mathrm{S}}}$. Thus, it follows that $C\left(S, Q^{G}\right) \geq C\left(S, \mathrm{Q}^{\mathrm{G}^{\mathrm{S}}}\right)$. Combining with the previous result, we obtain $C\left(S, Q^{G}\right) \geq \omega(G[S])=\chi(G[S])$.

Let $\left\{S_{1}, \ldots, S_{\omega(G[S])}\right\}$ be a partition of $S$ that solves the minimum coloring problem for $S$. By definition of $Q^{G}$, if $i, j \in S_{r}$ for some $r=1, \ldots, \omega(G[S])$, then $\{i, j\} \notin G$ and there is no $t \in M$ such that $q_{i t}^{G}=q_{j t}^{G}=1$. Thus, consider the network $z^{S}$ such that for all $r$, we randomly pick an agent $i_{r}$ in $S_{r}$, and build a capacity of 1 from $i_{r}$ to the source as well as from all other members of $S_{r}$ to $i_{r}$. This is a feasible network, and it is obvious that $\sum_{e \in E} z_{e}^{S} c_{e}=\sum_{i \in N} z_{i 0}^{S}=\omega(G[S])$. Thus, $C\left(S, Q^{G}\right) \leq \omega(G[S])$.

Combining, we obtain that $C\left(S, Q^{G}\right)=\omega(G[S])=C^{M}(S, G)$ for all $S \subseteq N$.

\section{A.5 Lemma 10}

Proof. Let $\left(N, M, Q^{G}, c\right)$ be as above and consider $C\left(N, Q^{G}\right)$. Since $G$ is a weakly perfect graph, $\chi(G)=\omega(G)$, and by the same logic as in the previous lemma, $C\left(N, Q^{G}\right)=\omega(G)=C^{M}(N, G)$.

Fix $S \subset N$ and let $\left\{S_{1}, \ldots, S_{\chi(G[S])}\right\}$ be a partition of $S$ that solves the minimum coloring problem for $S$. By definition of $Q^{G}$, if $i, j \in S_{r}$ for some $r=1, \ldots, \chi(G[S])$, then $\{i, j\} \notin G$ and there is no $t \in M$ such that $q_{i t}^{G}=q_{j t}^{G}=1$. Thus, we can build a path of capacity 1 connecting all members of $S_{r}$ to each other, with one agent connected to the source. By the definition of $c$, the cost of such a path is 1 . Thus, $C\left(S, Q^{G}\right) \leq \chi(G[S])=C^{M}(S, G)$.

Since $\left(N, M, Q^{G}, c\right)$ is an MSP problem, Core $(C)$ is non-empty. Since $C\left(S, Q^{G}\right) \leq C^{M}(S, G)$ for all $S \subset N$ and $C\left(N, Q^{G}\right)=C^{M}(N, G)$, Core $(C) \subseteq \operatorname{Core}\left(C^{M}\right)$. Thus, Core $\left(C^{M}\right)$ is non-empty. 